\title{
Machine Perfusion: Cold versus Warm, versus Neither. Update on Clinical Trials
}

\author{
E. Bonaccorsi-Riani, MD, PhD ${ }^{1,2, *} \quad$ I.M.A. Brüggenwirth, BS ${ }^{3, *} \quad$ J.E. Buchwald, $\mathrm{BS}^{4}$ S. lesari, $\mathrm{MD}^{2,5}$ \\ P.N. Martins, MD, PhD 4
}

${ }^{1}$ Abdominal Transplant Unit, Cliniques Universitaires Saint-Luc, Université catholique de Louvain, Brussels, Belgium

2 Pôle de Chirurgie Expérimentale et Transplantation, Université Catholique de Louvain, Brussels, Belgium

${ }^{3}$ Department of Surgery, University Medical Center Groningen, University of Groningen, Groningen, The Netherlands

${ }^{4}$ Division of Transplant, Department of Surgery, UMass Memorial Medical Center, University of Massachusetts, Worcester,

Massachusetts

${ }^{5}$ Department of Biotechnological and Applied Clinical Sciences, University of L'Aquila, L'Aquila, Italy

\begin{abstract}
Address for correspondence Paulo N. Martins, MD, PhD, FAST, FEBS FACS, Division of Transplant, Department of Surgery, UMass Memorial Medical Center, University of Massachusetts, 55 Lake Avenue, Worcester, MA 01655 (e-mail: paulo.martins@umassmemorial.org).
\end{abstract}

Semin Liver Dis 2020;40:264-281.

\begin{abstract}
Keywords

- ex situ machine perfusion

- liver transplantation

- extended criteria donors

- outcomes

- clinical trials

- organ preservation

Machine perfusion (MP) preservation is potentially one of the most significant improvements in the field of liver transplantation in the last 20 years, and it has been considered a promising strategy for improved preservation and ex situ evaluation of extended criteria donor (ECD) organs. However, MP preservation adds significant cost and logistical considerations to liver transplantation. MP protocols are mainly classified according to the perfusion temperature with hypothermic machine perfusion (HMP) and normothermic machine perfusion (NMP) being the two categories most studied so far. After extensive preclinical work, MP entered the clinical setting, and there are now several studies that demonstrated feasibility and safety. However, because of the limited quality of clinical trials, there is no compelling evidence of superiority in preservation quality, and liver MP is still considered experimental in most countries. MP preservation is moving to a more mature phase, where ongoing and future studies will bring new evidence in order to confirm their superiority in terms of clinical outcomes, organ utilization, and cost-effectiveness. Here, we present an overview of all preclinical MP studies using discarded human livers and liver MP clinical trials, and discuss their results. We describe the different perfusion protocols, pitfalls in MP study design, and provide future perspectives. Recent trials in liver MP have revealed unique challenges beyond those seen in most clinical studies. Randomized trials, correct trial design, and interpretation of data are essential to generate the data necessary to prove if MP will be the new gold standard method of liver preservation.
\end{abstract}

Over the past five decades, liver transplantation (LT) has become the standard of care for end-stage liver diseases. This success led the liver transplant community to broaden indications for LT. ${ }^{1,2}$ The direct consequence of this progress

\footnotetext{
These authors contributed equally to this work.
}

is the increasing number of patients on the waiting list, which worsens the chronic problem of organ shortage. In fact, the amount of LTs performed is directly affected by allograft availability, as reflected in the 10 to $20 \%$ mortality rate on the waiting list. ${ }^{3,4}$ To overcome this problem, transplant surgeons are required to accept allografts from published online June 18, 2020

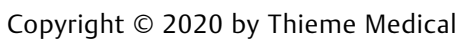
Publishers, Inc., 333 Seventh Avenue, New York, NY 10001, USA. Tel: +1(212) 760-0888. ISSN 0272-8087. 
donors with extended criteria (ECD), such as elderly donors, obese donors who regularly provide steatotic grafts, and donors after cardiac death (DCD). It is well known that these organs do not tolerate long periods of static cold storage (SCS), the current standard of liver graft preservation, as they are more susceptible to ischemia and reperfusion injury (IRI), have a higher incidence of primary nonfunction (PNF), and are more prone to develop biliary complications, which leads to a greater number of graft failures with a higher rate of retransplantation compared with non-ECD organs. ${ }^{5}$ Ex situ organ machine perfusion (MP) can be an alternative preservation modality to improve the quality and function of ECD allografts. The concept of ex situ organ perfusion, idealized by Charles Lindbergh and Alexis Carrel, who built the first MP prototype in 1935, 6,7 was abandoned after few experiments in the late 1960s performed in the United States and Europe ${ }^{8,9}$ due to improved static cold preservation solutions. ${ }^{10,11}$ The growing demand for ECD organs refueled the interest of the transplant community in MP preservation of ex situ allografts. In the past decade, MP has become a hot topic in organ transplantation and research groups worldwide have investigated different MP modalities to optimize preservation. ${ }^{12-14}$ In this current review, we present an overview of all clinical liver MP trials and MP studies using discarded human liver grafts. We analyzed recent clinical experience with liver MP, taking into account the different protocols with respect to tem- perature, type of perfusion, duration of perfusion, and postLT outcome.

\section{Types of Perfusion}

Ex situ liver perfusion protocols can be mainly defined according to the perfusion temperature used (-Fig. 1). In hypothermic machine perfusion (HMP) protocols, the temperature is maintained at 4 to $10^{\circ} \mathrm{C}$, while it is around 20 to $30^{\circ} \mathrm{C}$ in subnormothermic MP studies. In normothermic machine perfusion (NMP), the temperature is kept at physiological levels $\left(37^{\circ} \mathrm{C}\right) .^{15}$ Innovative modalities of organ preservation and perfusion include super cooling organ preservation $\left(-4^{\circ} \mathrm{C}\right)$ and hyperthermic perfusion $\left(>37^{\circ} \mathrm{C}\right)$. However, such strategies are still in the experimental stage. ${ }^{16-18}$ While HMP can be performed with or without active oxygenation, NMP always requires oxygenation. Other protocol variations include the perfusion route (through the hepatic artery, portal vein, or both vessels) and the number and kind of pumps (pulsatile vs. nonpulsatile) or via gravitational force like the portal vein perfusion in the OrganOx metra (OrganOx Limited) device.

\section{Hypothermic Machine Perfusion}

For years, cooling has been the cornerstone in organ preservation. The concept of hypothermic preservation is largely

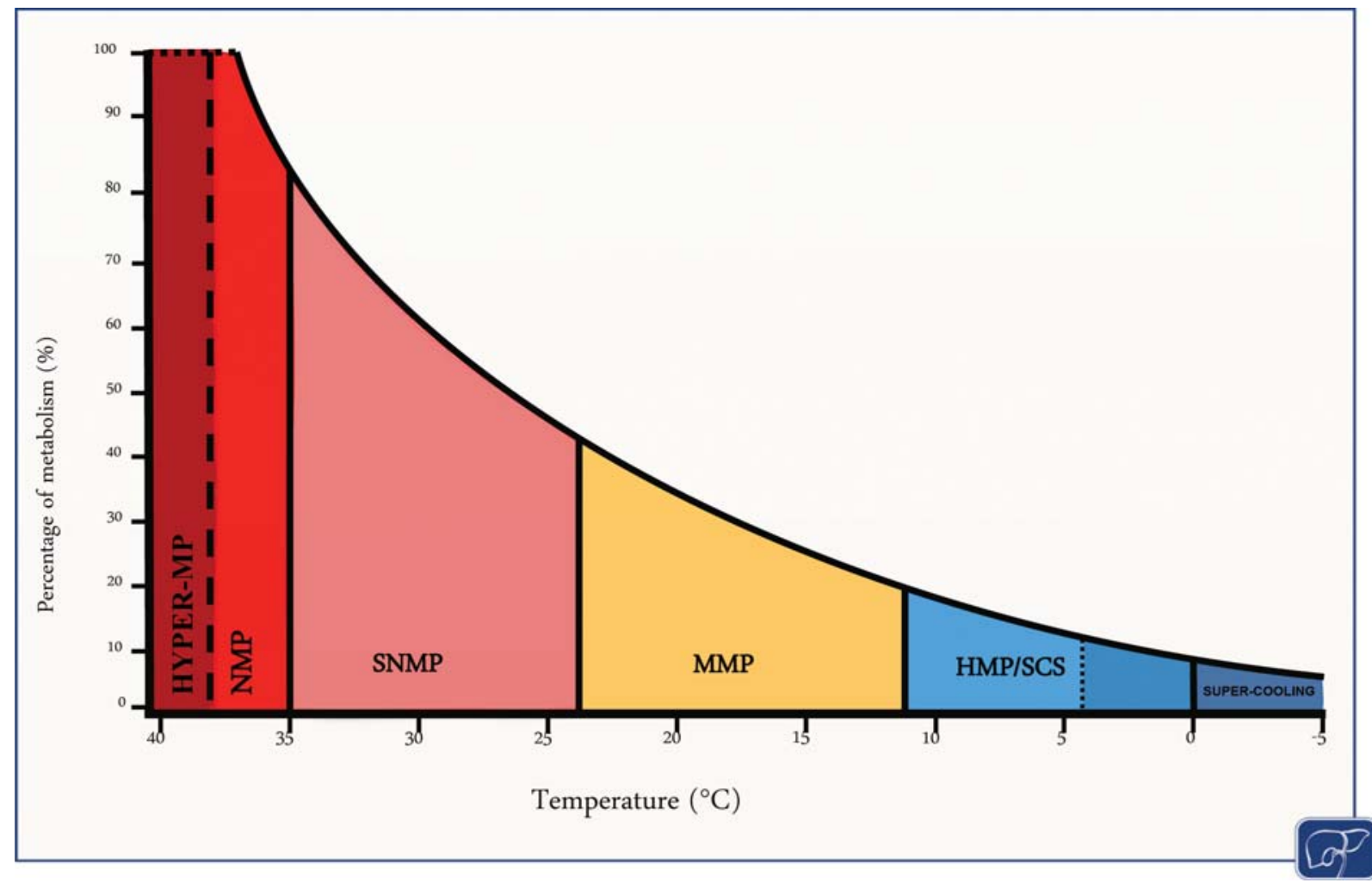

Fig. 1 Graph representing the dynamics of the metabolism rate according to the machine perfusion temperature used. Based on Van't Hoff's principle (expressed as $\left.\mathrm{Q}_{10}=(\mathrm{k} 2 / \mathrm{k} 1)^{10 /(\mathrm{t} 2 \mathrm{-t} 1)}\right)$ the metabolism rate is much lower in the cold $\left(0-12^{\circ} \mathrm{C}\right)$. Types of organ preservation and machine perfusion proposed in accordance with temperature intervals: Hyper MP, hyperthermic machine perfusion $\left(38-42^{\circ} \mathrm{C}\right)$; NMP, normothermic machine perfusion $\left(35-38^{\circ} \mathrm{C}\right)$; SNMP, subnormothermic machine perfusion $\left(24-34^{\circ} \mathrm{C}\right)$; MMP, midthermic machine perfusion $\left(13-24^{\circ} \mathrm{C}\right)$; $\mathrm{HMP}$, hypothermic machine perfusion $\left(0-12^{\circ} \mathrm{C}\right)$; SCS, static cold storage $\left(0-8^{\circ} \mathrm{C}\right)$, super-cooling preservation $\left(-4\right.$ to $\left.0^{\circ} \mathrm{C}\right)$. (Adapted from Karangwa et al. ${ }^{15}$ ) 
based on the idea that the graft is maintained in a hypometabolic state, thereby decreasing energy utilization during ischemia and preserving essential mechanisms to regenerate ATP upon reperfusion. However, due to persistent low-grade cellular metabolism at $4^{\circ} \mathrm{C}$, SCS preservation on ice leads to anaerobic metabolism within short time due to the lack of oxygen and substrates. In contrast, the concept of HMP is to maintain viability and aerobic metabolism prior to implantation of the graft by providing oxygen while the organ's metabolism is limited. ${ }^{19}$ The underlying mechanisms in HMP mainly involve resuscitation of mitochondria. Even a short period of 2 hours of oxygenated HMP can switch mitochondria from ischemic to fully recharged cellular energy levels. ${ }^{19,20}$

MP was traditionally designed as a continuous approach, starting directly after organ procurement. However, in the last decade, it became clear that end-ischemic (after SCS) MP is an attractive and simpler approach, avoiding transportation of the perfusion devices and extra perfusion personnel to the donor hospital. Livers are preserved by SCS after procurement and are then subjected to end-ischemic MP upon arrival at the recipient center. Even after relatively longer periods of SCS, end-ischemic HMP can protect liver grafts. $^{21}$ An additional advantage of HMP is that in case of pump failure, the liver can easily be restored to traditional SCS. Also, since the organ is in a hypometabolic state, it produces lesser waste products and requires less intensive labor by perfusionists when compared with NMP. A disadvantage of HMP used to be the lack of reliable prediction methods of organ function. However, a recent study by Muller et al shows that real-time fluorometric analysis of perfusate flavin, a marker of mitochondrial injury, can be predictive of graft function and loss during cold MP. ${ }^{22}$ This achievement has high clinical relevance, and real-time estimation of liver function during cold preservation could substantially increase safe utilization of liver grafts.

\section{Prospective Clinical Studies Using Hypothermic Machine Perfusion}

In 2010, Guarrera et al were the first to report successful transplantation of 20 ECD donation after brain death (DBD) human livers after HMP via the portal vein and hepatic artery without active oxygenation ( - Table $\mathbf{1}$ ) ${ }^{23}$ Because this was a nonrandomized trial comparing a series of machine-perfused livers with historical controls, they were only able to demonstrate safety of end-ischemic HMP of human liver grafts during 3 to 7 hours. This was also the first study to describe that the HMP system is user-friendly, such as that MP pressures and temperature remained stable, and little intervention from the perfusionist was needed. They found diminished peak aspartate aminotransferase (AST) and alanine aminotransferase (ALT), shorter hospital length of stay, and fewer biliary complications in the HMP-treated grafts compared with the matched SCS group. In the years following, HMP was increasingly used in kidney transplantation worldwide, but clinical implantation in LT remained investigational. ${ }^{24}$ Five years after publishing their first clinical series, Guarrera et al reported successful transplantation of
31 "orphan" ECD livers after preservation by HMP in a nonrandomized fashion. ${ }^{25}$ The term orphan was denoted to any liver turned down by all centers within the donor's originating United Network for Organ Sharing region, requiring more complex allocation management and expected longer cold ischemia time (CIT). Their study shows good outcomes with $84 \%$ graft survival 1 year after transplantation. In addition, biliary complications, often described as the "Achilles heel" of LT, occurred significantly less in the HMP group compared with the SCS historical control group. Better preservation of the arterial vasculature lining the biliary tree by providing a continuous supply of oxygen, better distribution of the perfusion fluid, and washout of waste products is suggested to play a role. ${ }^{26}$ In the same year, Dutkowski et al reported on a nonrandomized study of 25 DCD livers treated by oxygenated HMP (HOPE) through the portal vein only. ${ }^{27}$ Lower peak ALT, lesser cases of intrahepatic cholangiopathy and biliary complications, and 1-year graft survival of $90 \%$ versus $69 \%$ were observed in the HOPE group compared with the SCS historical control group. Although conclusive evidence of a beneficial effect of HMP on reducing biliary injury after transplantation could not yet be provided, these first results seemed promising. In 2017, van Rijn and colleagues published a nonrandomized clinical study of end-ischemic dual HOPE (DHOPE) in 10 DCD liver grafts. Compared with SCS alone, DHOPE seemed to provide better preservation of DCD liver grafts, resulting in reduced graft injury and improved early graft function. Noteworthy, none of the 10 DHOPE-preserved livers required retransplantation for nonanastomotic biliary strictures (NAS), compared with 5 out of 20 in the control group. ${ }^{28}$ The potential benefit of DHOPE to better preserve the biliary tree is investigated in a randomized clinical trial (RCT), initiated by Groningen et al in 2015 (clinicaltrials.gov; NCT02584283) and of which the results are expected mid-2020. In collaboration between liver transplant centers in the Netherlands, the United Kingdom, and Belgium, this trial investigates the impact of DHOPE preservation versus SCS in DCD liver grafts. The primary outcome of the DHOPE-DCD trial is the development of NAS within 6 months after LT. Single HOPE is investigated by the prospective HOPE-ECD-DBD clinical trial started in 2017 by the University of Aachen. In this RCT using DBD livers only, 1 to 2 hours of single HOPE preservation is compared with the traditional SCS (clinicaltrials.gov; NCT03124641).

The results of two single-center trials from France and Italy may be expected this year. The PIO trial from Italy compares 10 ECD livers treated with HOPE with 30 matched historical controls preserved by SCS (clinicaltrials.gov; NCT03031067). The PERHO trial from France compares 25 DBD livers preserved by HOPE with 75 historical controls preserved by SCS (NCT03376074). The results of several other clinical trials may be expected in the coming years. As such, a large RCT in the United States is investigating HMP versus SCS in 140 transplant recipients with the incidence of early allograft dysfunction (EAD) as the primary outcome (NCT03484455). The multicenter HOPExt trial from France randomizes 266 ECD liver grafts between HOPE and SCS, also studying EAD as its primary outcome (NCT03929523). Similarly, an RCT in France 


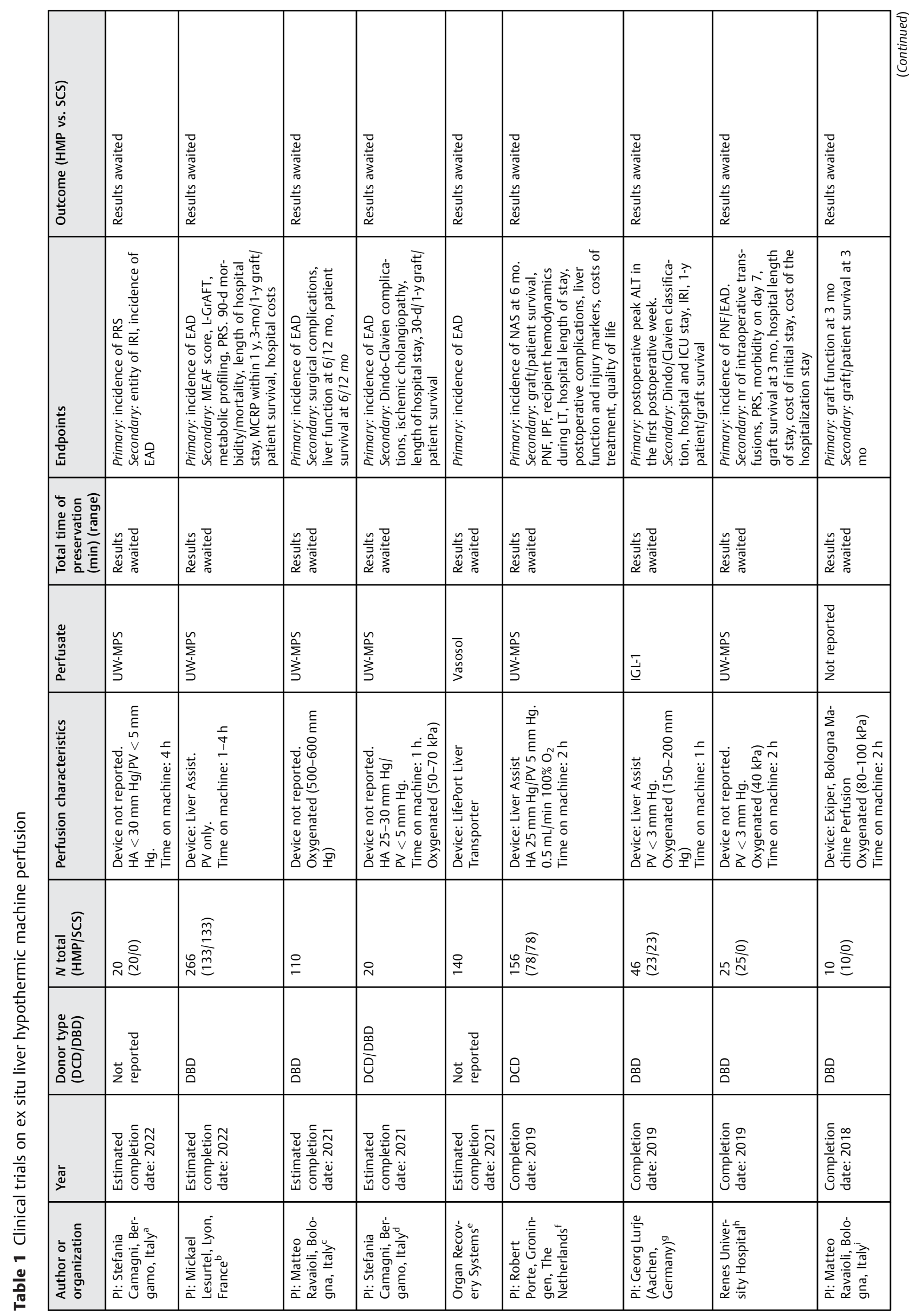




\begin{tabular}{|c|c|c|c|c|}
\hline 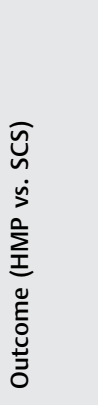 & 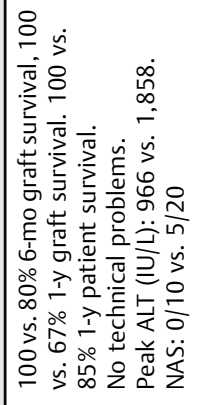 & 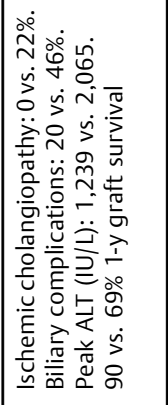 & 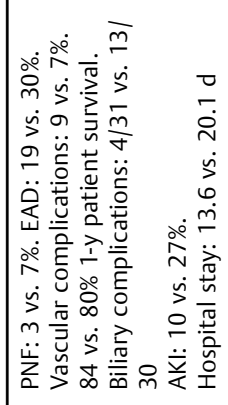 & 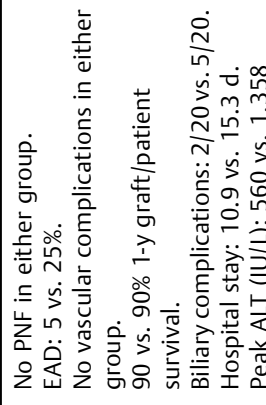 \\
\hline 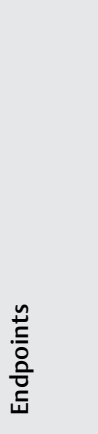 & 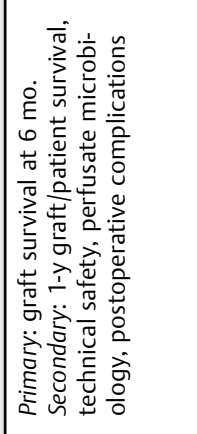 & 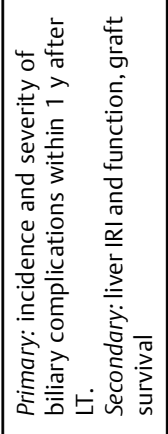 & 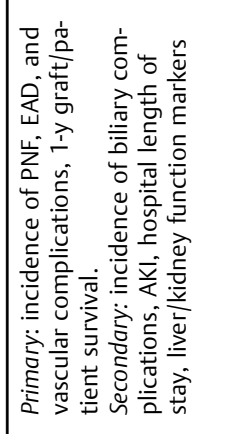 & 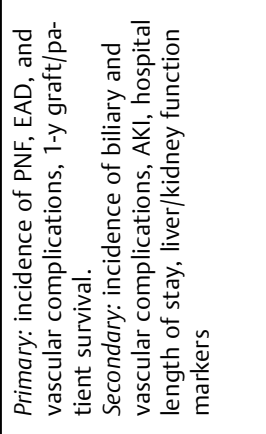 \\
\hline 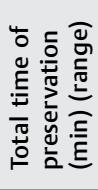 & 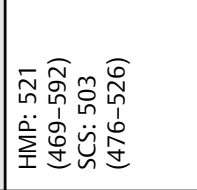 & 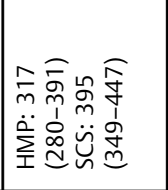 & 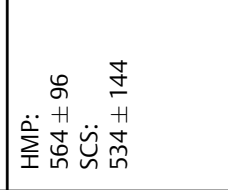 & 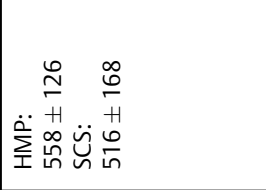 \\
\hline 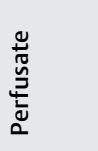 & $\sum_{j}^{n}$ & $\begin{array}{l}\overline{\check{n}} \\
\underline{\underline{n}}\end{array}$ & 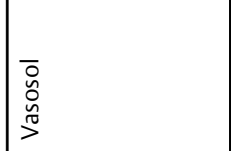 & \begin{tabular}{l}
$\bar{o}$ \\
$\overline{0}$ \\
$\tilde{o}$ \\
\multicolumn{0}{c}{}
\end{tabular} \\
\hline 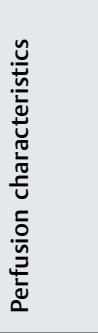 & 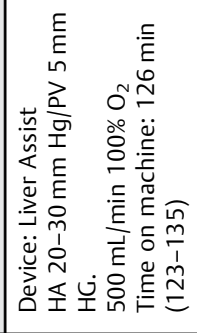 & 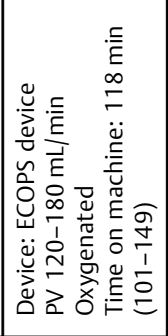 & 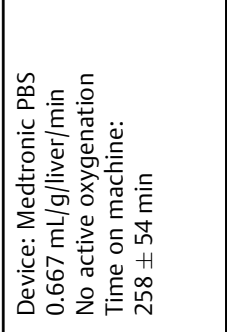 & 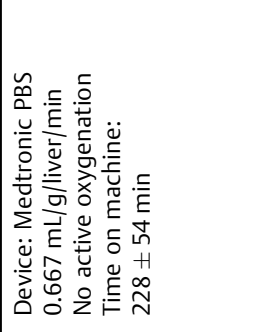 \\
\hline 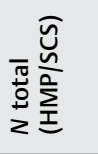 & $\underset{m}{\stackrel{\widehat{D}}{\stackrel{D}{O}}}$ & 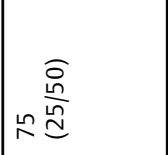 & 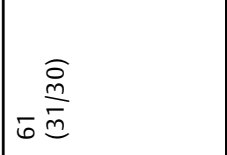 & \& \\
\hline 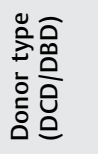 & $\begin{array}{l}\text { 응을 } \\
\text { 에 } \\
\text { 产 } \\
\text { 品 }\end{array}$ & 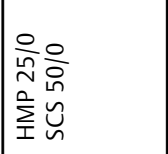 & 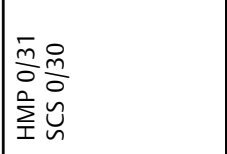 & 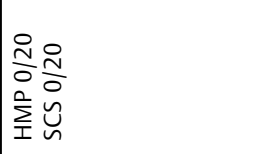 \\
\hline 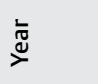 & 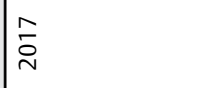 & $\stackrel{n}{\stackrel{n}{\sim}}$ & $\stackrel{n}{\stackrel{n}{\sim}}$ & $\stackrel{\circ}{\stackrel{\sim}{\sim}}$ \\
\hline 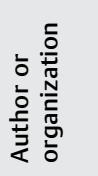 & 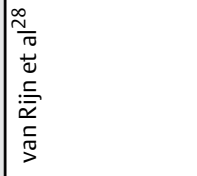 & 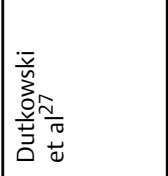 & 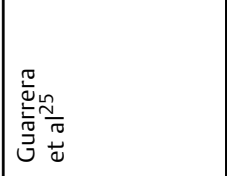 & 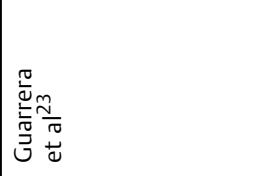 \\
\hline
\end{tabular}

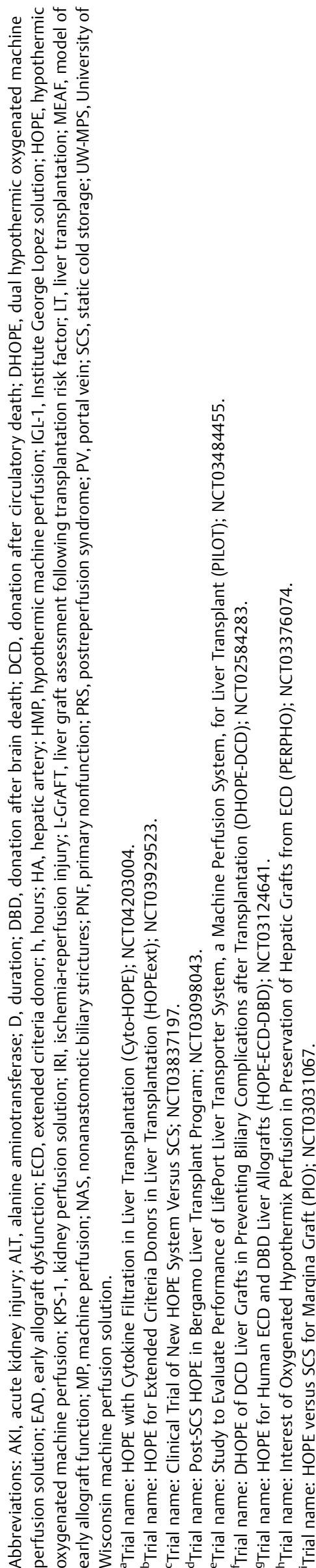


compares HOPE versus SCS in 110 ECD livers with EAD as the primary outcome (NCT03837197). A smaller single-center study from the Papa Giovanni XXIII Hospital in Italy aims to study the feasibility and safety of HOPE in their liver transplant program in 20 patients (NCT03098043). In 2020, the same hospital plans to start a RCT to study the effects of HOPE using a cytokine filter (cyto-HOPE) versus traditional HOPE in 20 ECD livers (NCT04203004). The primary outcome of the trial is the incidence of post-reperfusion syndrome in the recipient, hypothesizing that cytokine filtration further reduces IRI during HOPE.

\section{Hypothermic Machine Perfusion in Experimental Studies Using Discarded Human Livers}

The majority of studies published on HMP over the last decades involve mainly preclinical studies without implantation of the graft. Instead, reperfusion with whole blood and reoxygenation is used to simulate transplantation. Most experimental studies have used animal models to investigate different HMP modalities, and to date, only a handful of studies using declined human livers have been published (-Table 3 ).

There is a wide variance in the reported HMP perfusion times. In general, HMP perfusion protocols are shorter than for NMP. Perfusion durations up to 7 hours have been reported in clinical studies, but a period of 1 to 2 hours of end-ischemic HMP is most commonly used. ${ }^{23,25,27-29}$ Cold oxygenation restores cellular energy levels already within the first 1 to 2 hours of perfusion. ${ }^{19,29,30}$ In addition, a short period of 2 hours of HMP seems to improve endothelial cell function as evidenced by increased nitric oxide production upon reperfusion of declined DCD livers, while this increase was not observed in SCS-preserved grafts. ${ }^{31}$ Vekemans et al found reduced levels of endothelin-1 (a vasoconstrictive factor) and intercellular adhesion molecule-1 (an inflammatory factor) after HMP preservation compared with SCS, suggesting a potential benefit of HMP on vascular hemostasis and endothelial cell stability. However, the effect did not persist during reperfusion, making it unlikely to have an effect in a real transplant setting. ${ }^{29}$ Besides short-term preservation, HMP holds potential to extend liver preservation times, which could facilitate logistics for allocation and transplantation. De Carlis et al recently reported successful transplantation of two livers with extended CIT for up to 20 hours including 8-hour end-ischemic HMP. ${ }^{21}$ In addition, Brüggenwirth et al showed successful preservation of porcine and discarded human livers after 24-hour DHOPE with similar hepatocellular function and injury compared with shorter preservation times. It would be of interest to further investigate the boundaries of extended cold MP. ${ }^{32}$

Several studies have been undertaken to elucidate the beneficial effects of oxygenated versus nonoxygenated HMP. In 2005, the first study describing HMP in discarded human livers was published by Guarrera et al, which showed safe preservation of livers without active oxygenation of the MP solution. ${ }^{33}$ The work of Schlegel et al has shown that complete absence of oxygen during HMP triggers hepatocyte cell death via mitochondria, and fails to prevent reperfusion injury. ${ }^{19}$ The liver is a metabolically high-demanding organ, even under hypothermic conditions. Therefore, the dissolved oxygen in the perfusate may be sufficient for standard grafts, but active oxygenation is desired in grafts with more anticipated preservation injury, such as DCD livers. ${ }^{34}$ The two clinical trials performed by Guarrera et al showed good results after HMP without active oxygenation in DBD livers. ${ }^{23,25}$ Both clinical trials using DCD livers were performed using an oxygenated MP solution. ${ }^{27,28}$ A recent preclinical study by Abudhaise et al failed to observe differences between oxygen-supplemented versus non-oxygen-supplemented portal venous perfusion during HMP in DCD and DBD livers. ${ }^{35}$ However, alterations between the groups are most likely to manifest after rewarming and reperfusion with whole blood, which was not performed in their study. Monbaliu et al performed HMP without active oxygenation in DBD and DCD liver grafts and they reported signs of hypoxic injury as reflected by the presence of anoxic vacuoles on liver electron microscopy images, suggesting that supplemental oxygenation might have been beneficial. ${ }^{36}$ On the other hand, some researchers fear the effects of oxidative stress when the liver is exposed to oxygen under cold conditions. However, several studies demonstrate only minimal oxidative stress during HOPE, even when high oxygen concentrations are used, or when HOPE is extended for longer hours. ${ }^{19,32,37}$

Caution should be given to the perfusion devices' pressure settings during HMP, as higher vascular resistances in the cold pose a risk of undesired endothelial shear stress to the liver sinusoids. ${ }^{38}$ Some of the currently used perfusion devices are flow-controlled, such as the Medtronic PBS used by Guarrera et al, whereas others are pressure-controlled, like the Liver Assist from Organ Assist used by Porte et al. 23,25,28 Based on recent studies, adjusting MP settings to portal pressures of $\leq 3 \mathrm{~mm} \mathrm{Hg}$ and arterial pressures of $\leq$ $25 \mathrm{~mm} \mathrm{Hg}$ can avoid shear stress. ${ }^{19,39}$

Regarding the perfusion route, two different approaches have been advocated. Single portal vein perfusion adds simplicity and has been shown to mitigate IRI of the liver parenchyma and biliary tree. ${ }^{27}$ Complete perfusion of the liver and the biliary system during single portal vein perfusion was shown by fluorescence in rat, pig, and discarded human livers. ${ }^{40}$ However, researchers opting for a dual perfusion route suggest improved oxygen supply to the peribiliary vascular plexus of the bile ducts by additional arterial perfusion. ${ }^{26,41}$ In a feasibility study using discarded human livers, Jomaa et al found no effect of using a dual versus single MP approach on sinusoidal endothelial injury. ${ }^{42}$ On another note, arterial perfusion only does not seem to be a favorable approach. ${ }^{35}$ In clinical trials, Dutkowski et al have repeatedly used single HOPE, whereas Guarrera et al and van Rijn et al used dual HOPE in their series. ${ }^{23,25,27,28}$ Mitigating biliary IRI and reducing ischemic cholangiopathy remain a lofty goal for DCD LT in particular. It remains unknown whether dual perfusion is superior to prevent ischemic cholangiopathy after transplantation, and only randomized trials comparing a single versus dual approach could answer this question. Currently, the only clinical trial 
studying the effects of DHOPE uses DCD livers, whereas single HOPE is used in a prospective trial in DBD grafts. A recent report by van Leeuwen et al describes feasibility of HMP through the umbilical vein instead of the portal vein in discarded human livers. ${ }^{43}$ Portal venous flows were similar to those obtained after cannulation of the portal vein main stem. MP through the umbilical vein could enable continuous oxygenated perfusion of liver grafts during procurement, splitting, and implantation.

A variety of MP solutions have been explored during HMP, with Belzer MP solution, histidine-tryptophan-ketoglutarate preservation solution, Polysol, Celsior, Institut Georges Lopez preservation solution, and UW gluconate being the most routinely used. ${ }^{29}$ The majority of clinical perfusions are based on the original or modified UW solution. Solutions with low potassium concentrations and without the presence of starch appear particularly advantageous. A low potassium concentration decreases vascular resistance under hypothermic conditions and the addition of starch has been shown to increases perfusate viscosity. ${ }^{44}$ Under HMP, there is no need to add an oxygen carrier and the transport of oxygen is done passively though dilution.

\section{Normothermic Machine Perfusion}

In this MP preservation protocol, as the name implies, the temperature of the perfusate is maintained at "normal," physiological levels at $37^{\circ} \mathrm{C}$. The objective of mimicking the physiological environment is to keep the metabolic activity of the organ as close as possible to normal levels to minimize the impact of IRI, thereby increasing viability of the organ. To do this, NMP requires the use of oxygenators and the presence of oxygen transporters in the perfusate, with human red blood cells being the most common oxygen transporter used in current protocols. ${ }^{45-50}$ Unlike the HMP configuration, in NMP protocols, liver grafts are normally dually perfused through the portal vein and the hepatic artery. The maintenance of the organ metabolic activity during NMP offers the possibility to assess organ viability by measuring many metabolic parameters, such as lactate clearance, $\mathrm{pH}$ and glycemic levels, as well as bile production and its constitution. ${ }^{51}$ Finally, normothermic conditions seem to offer a more adequate setting for organ modulation, such as the use of defatting cocktails or gene therapies during ex situ perfusion.

\section{Prospective Clinical Studies Using Normothermic Machine Perfusion}

While two case reports were initially published online before 2016 describing LTs performed with ECD livers after preservation by NMP, the first multicenter clinical trial comparing NMP versus SCS was conducted by the Oxford group being published by Ravikumar et al in 2016 (- Table 2). ${ }^{45,52,53}$ In this nonrandomized study, which was designed to assess the viability and safety of NMP by comparing 20 LTs after NMP preservation with 40 LTs using SCS preservation (20\% of DCD grafts in each group), no differences were observed in 30-day graft and patient survival rates and in 6-month patient survival rates between the two groups. ${ }^{45}$ Later, two other non-RCTs conducted independently at two Canadian transplant centers, using the same Oxford perfusion device, confirmed the feasibility and safety of NMP. ${ }^{46,47}$ Selzner et al of the Toronto group compared 10 NMPpreserved livers with a matched historical series of 30 transplanted livers using SCS. The proportion of DCD livers was 20\% in the NMP group and $27 \%$ in the control group, and both groups presented similar graft and patient survival rates at 1 month, without differences in graft function in the early stages. ${ }^{46}$ The second clinical trial conducted at the University of Alberta, with a similar study design, confirmed the absence of differences in graft survival rates at 1 and 6 months after transplantation. It is worth noting that one DCD liver was discarded shortly after connection to the machine due to a hidden portal venous twist that prevented organ perfusion. ${ }^{47}$ In July 2014, the Oxford team initiated the first European multicenter RCT on NMP to extensively assess graft function and survival at 6 months and 1 year, as well as patient morbidity and mortality. This study, published in 2018, compared 121 patients who received an NMP-preserved liver with 101 patients transplanted with SCS-preserved livers. They showed lower rates of EAD and reperfusion syndrome. However, no differences were observed in the overall complication rates, hospital and intensive care unit length of stay, and in 1year graft and patient survival rates. Noteworthy, the NMP group had $28 \%$ of DCD grafts versus $21 \%$ in the SCS group, and with longer functional warm ischemia time and total preservation time compared with the SCS group. Furthermore, there was no significant difference in the rate of biliary complications (anastomotic and nonanastomotic strictures) between the two groups, neither for DCD nor DBD grafts at the MRCP performed at 6 months post-LT. ${ }^{48}$ Recently, the Pisa group conducted the first RCT to investigate the impact of NMP on LT with older donors. Twenty grafts from donors aged $\geq 70$ years were randomized in a 1:1 ratio to NMP or SCS preservation. There was no statistical difference in the patient and graft survival rates at 6 months. A patient in the NMP group received a second graft due to hepatic artery thrombosis 10 days after LT. On the other hand, one patient in the SCS group died 31 days after LT due to an episode of intestinal occlusion that evolved into sepsis shock. ${ }^{49} \mathrm{~A}$ single-center study from the Cleveland group tested the use of fresh-frozen plasma (FFP) as a constituent of the perfusate in association with RBC units. Using a noncommercial custom-made perfusion device, Liu et al compared the outcome of 21 LT performed with NMP-preserved livers to a historical series of LT performed with livers preserved by SCS in a 1:4 ratio. The NMP group presented less EAD, no cases of biliary complications, and the only death in this group was due to a cerebral vascular accident 8 months after LT. ${ }^{50}$ Recently, Jassem et al reanalyzed liver tissue samples and hepatic mononuclear cells collected from DBD livers transplanted after NMP and initially published by Ravikumar et al. They observed that NMP changed the gene expression profile from proinflammation to prohealing and regeneration compared with SCS liver samples. In addition, they observed a reduction in the population of $\mathrm{T}$ cells that produce inflammatory factors and an increase in the pool of regulatory T cells in NMP livers. These livers presented less necrosis and apoptosis and less neutrophil 


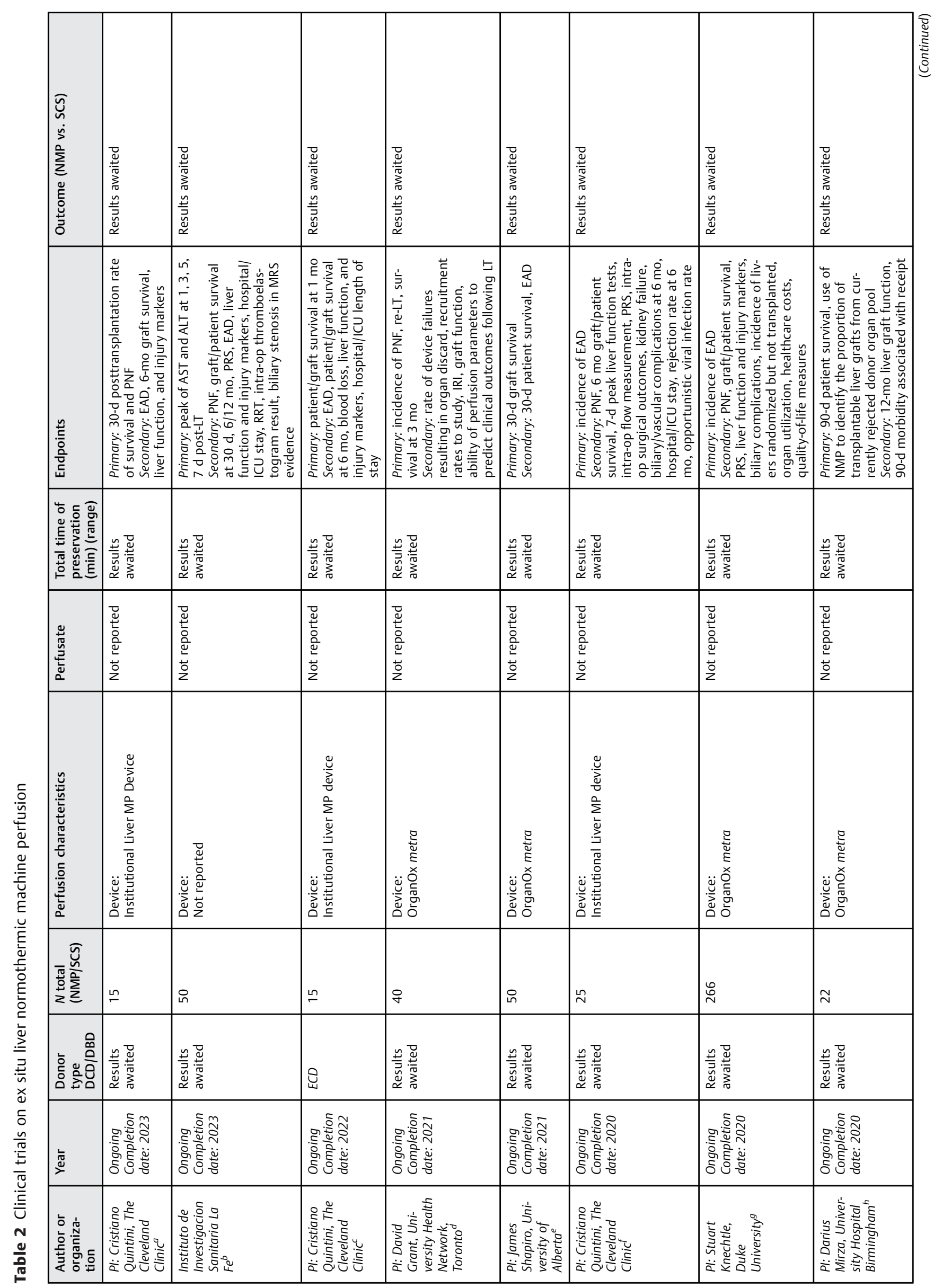




\begin{tabular}{|c|c|c|c|c|c|c|c|}
\hline 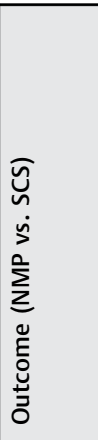 & & 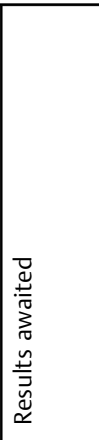 & 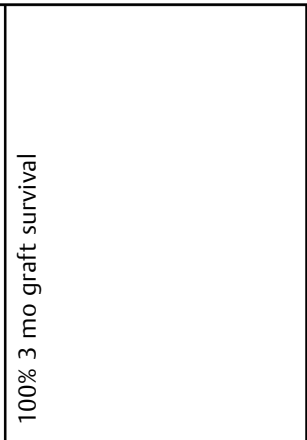 & 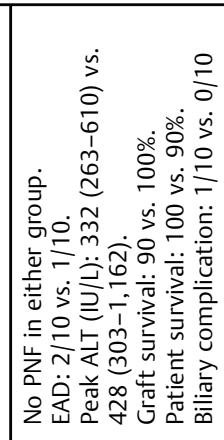 & 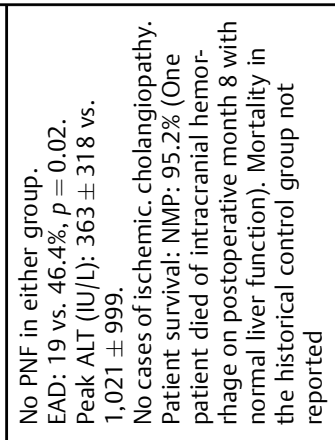 & 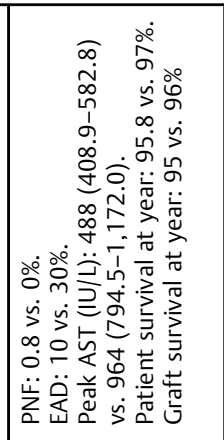 & 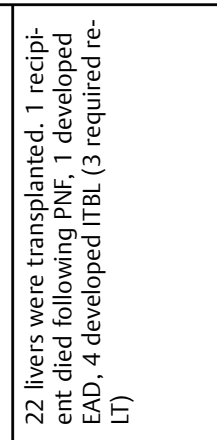 \\
\hline 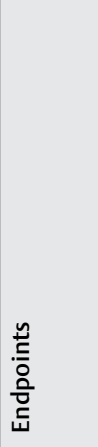 & 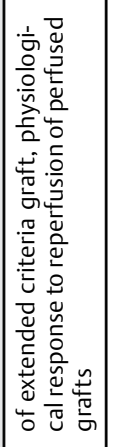 & 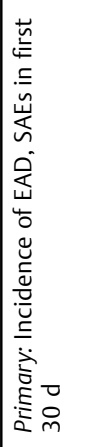 & 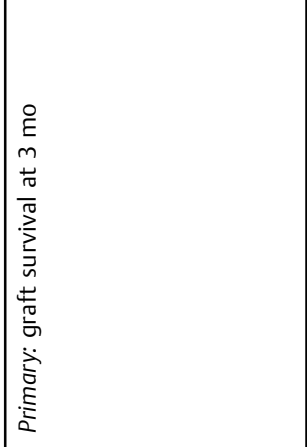 & 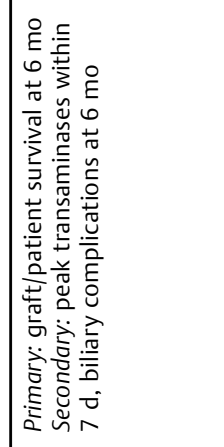 & 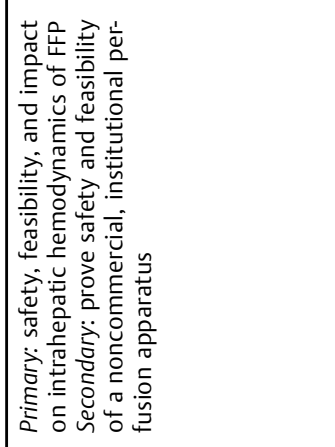 & 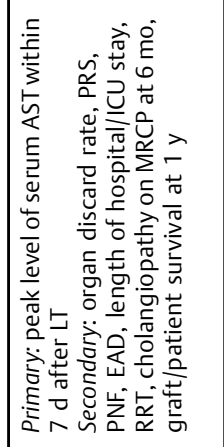 & 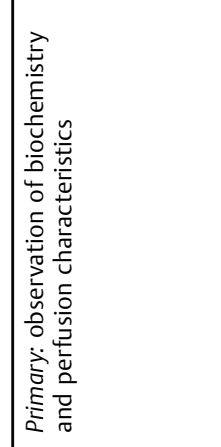 \\
\hline 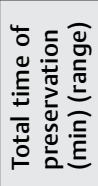 & & 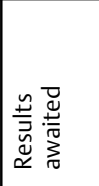 & 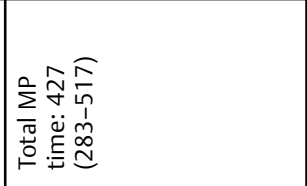 & 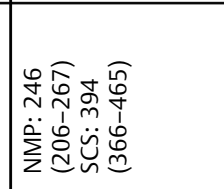 & 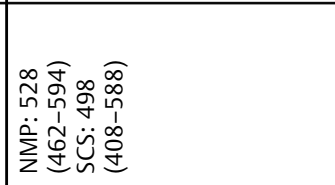 & 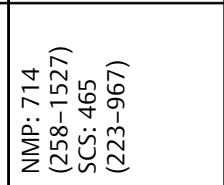 & 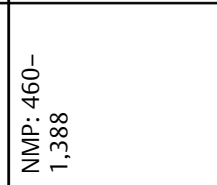 \\
\hline 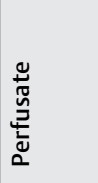 & & 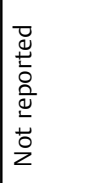 & 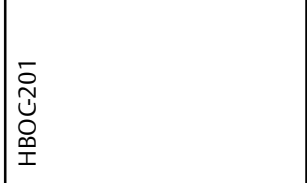 & 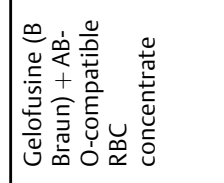 & 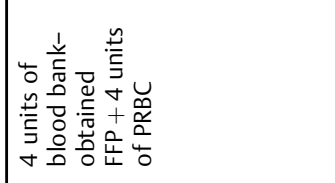 & 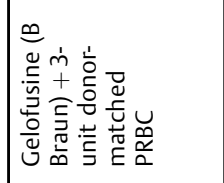 & 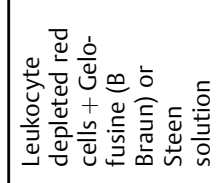 \\
\hline 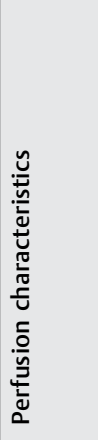 & & 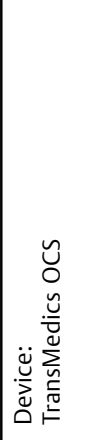 & 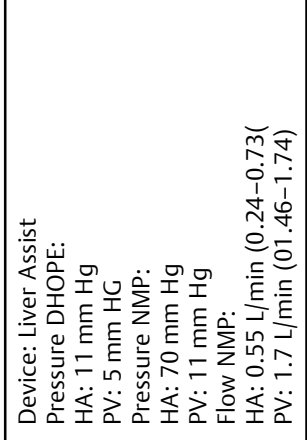 & 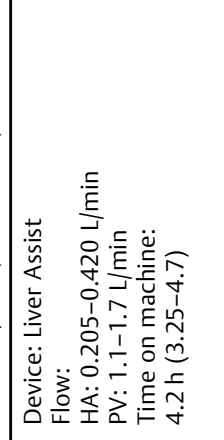 & 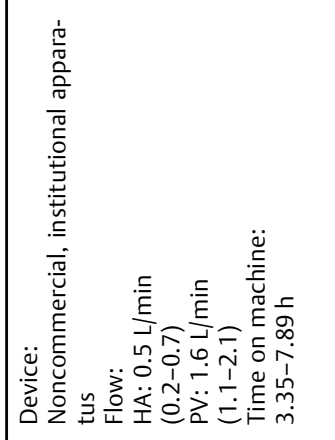 & 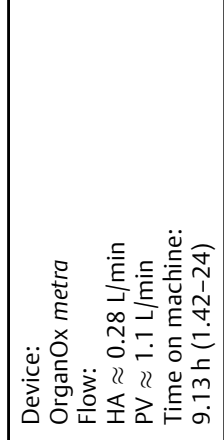 & 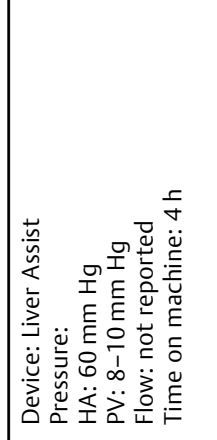 \\
\hline 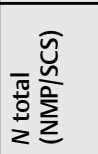 & & ஓे & $\frac{o}{\frac{a}{}}$ & 을 & 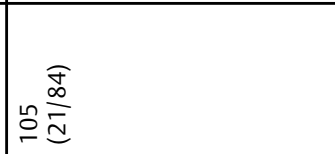 & 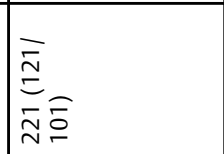 & 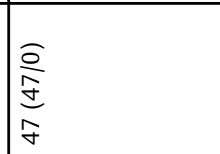 \\
\hline 竞 & & 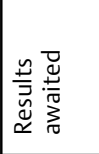 & 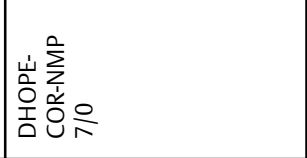 & 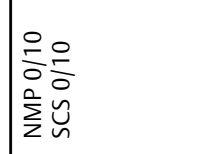 & 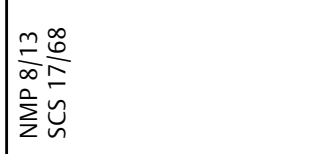 & 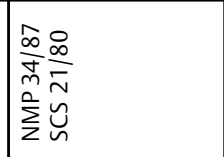 & 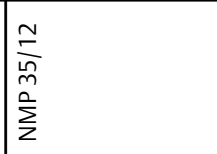 \\
\hline 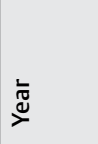 & & 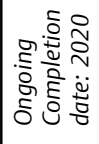 & 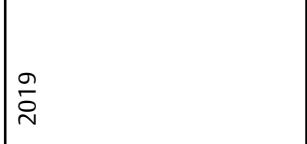 & 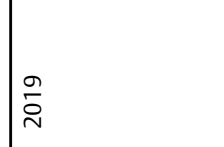 & $\frac{\mathfrak{r}}{\grave{N}}$ & $\stackrel{\infty}{\stackrel{\sim}{\sim}}$ & $\stackrel{\infty}{\stackrel{2}{~}}$ \\
\hline 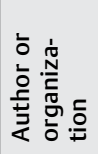 & & 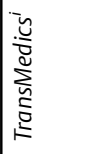 & 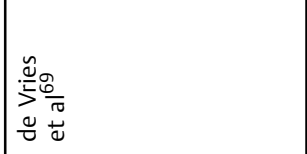 & 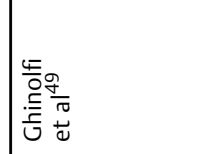 & 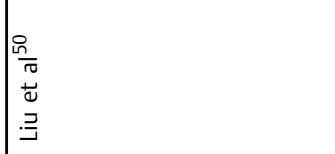 & 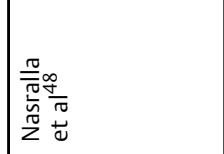 & 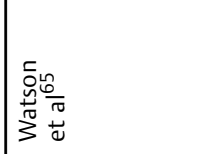 \\
\hline
\end{tabular}




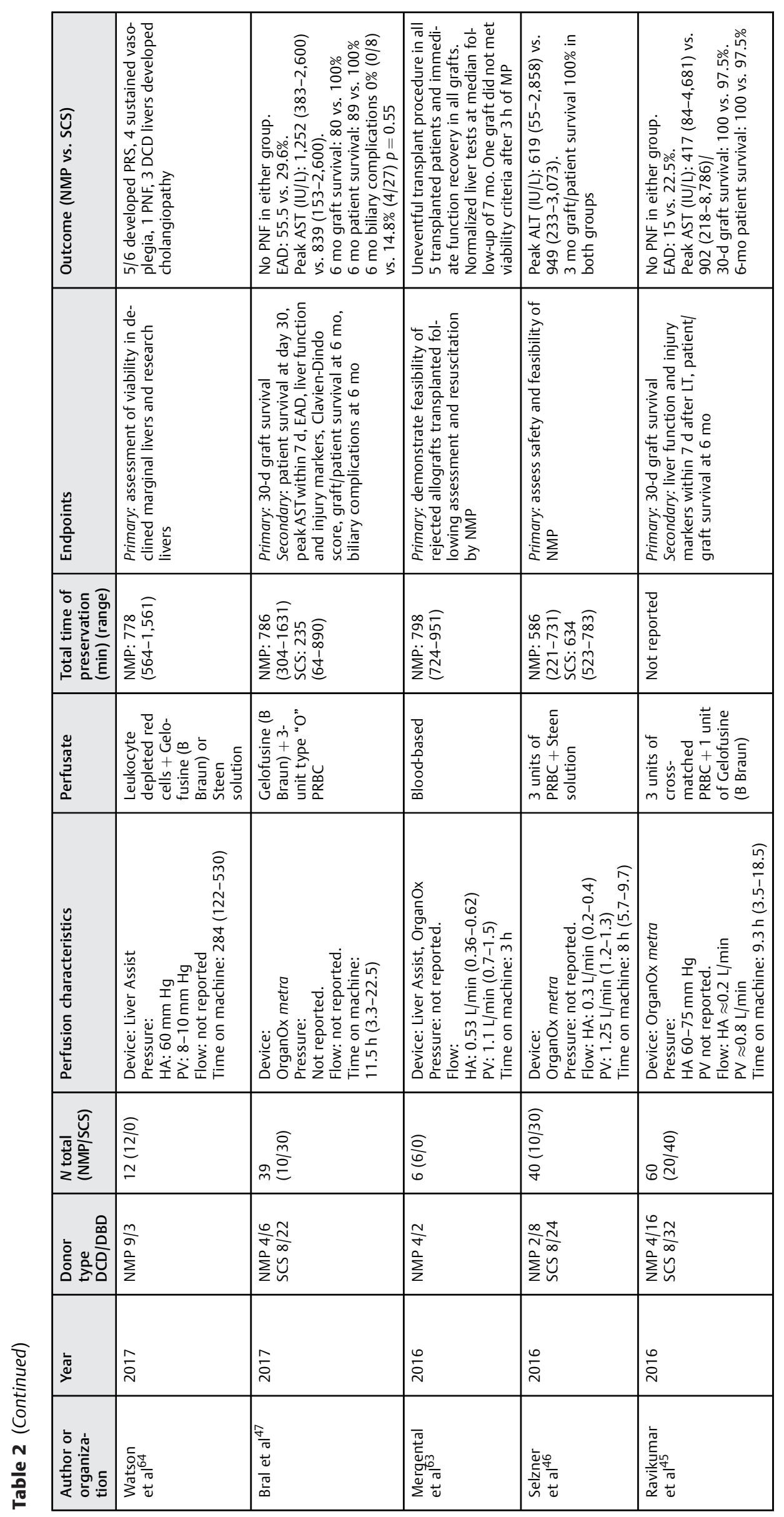

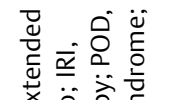

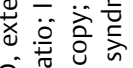

岂通这产

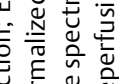

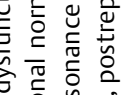

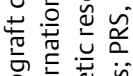

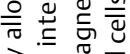

즌

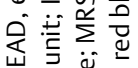

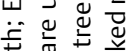

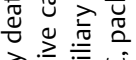

त्रें

.

至

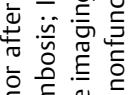

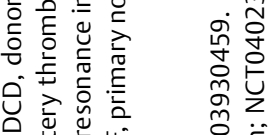

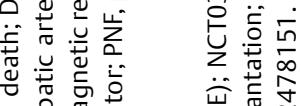

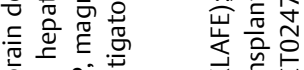

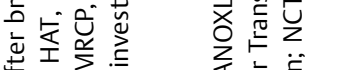

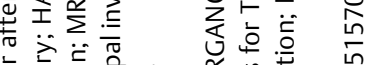

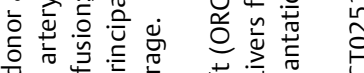

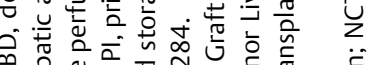

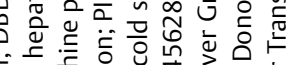

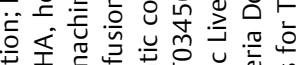

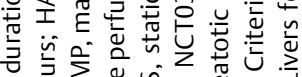

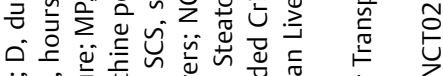

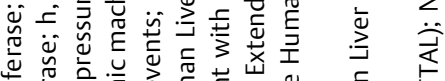

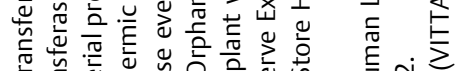

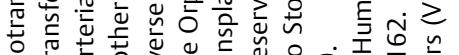

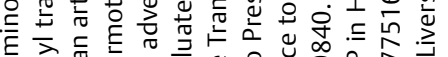

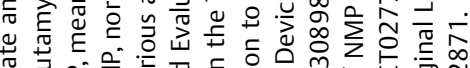

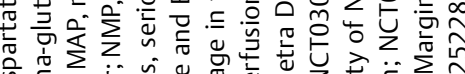

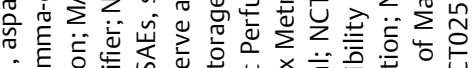

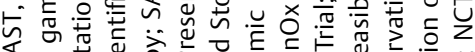

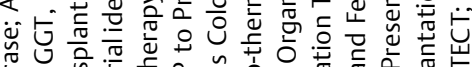

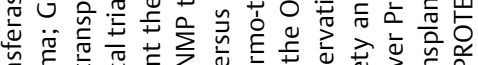

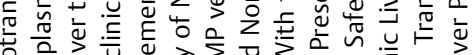

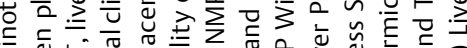

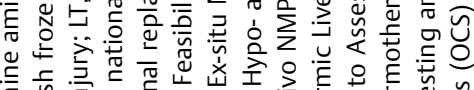

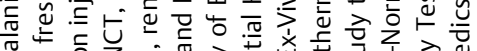

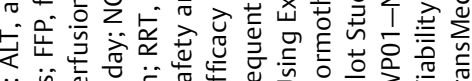

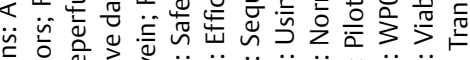

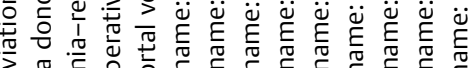
즌.

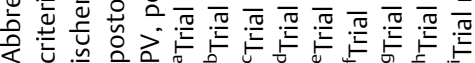




\begin{tabular}{|c|c|c|c|c|c|c|c|c|c|}
\hline 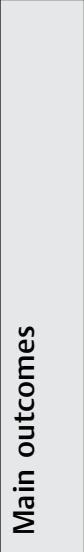 & & 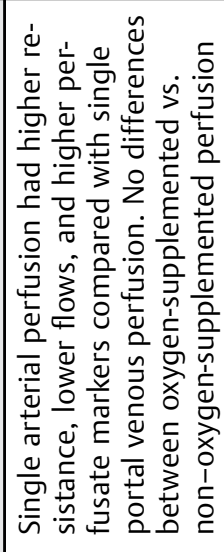 & 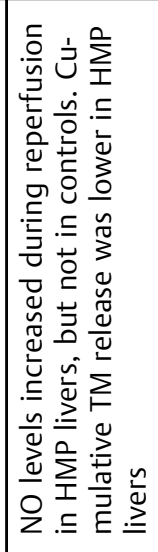 & 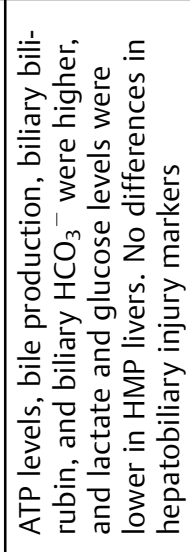 & 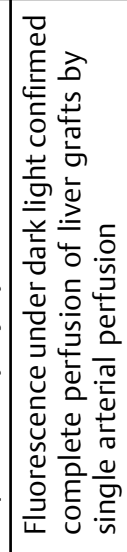 & 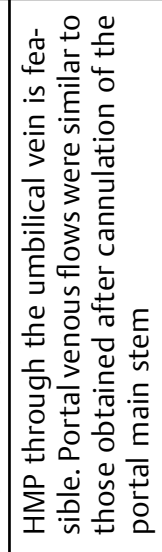 & 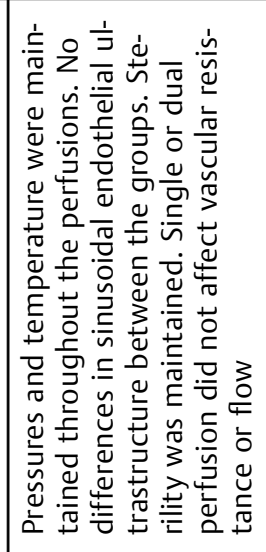 & 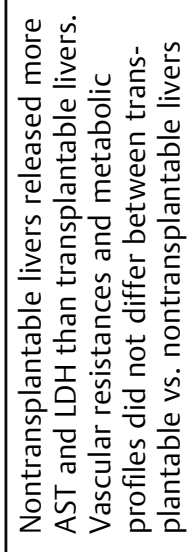 & 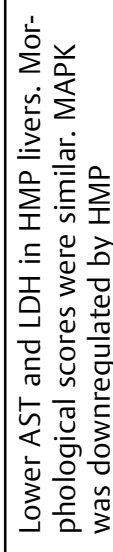 \\
\hline 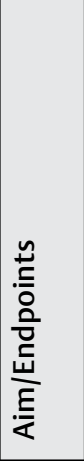 & & 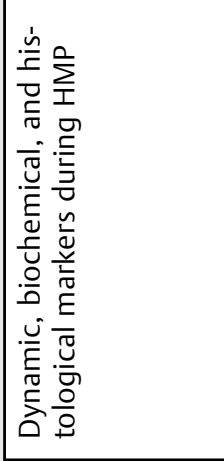 & 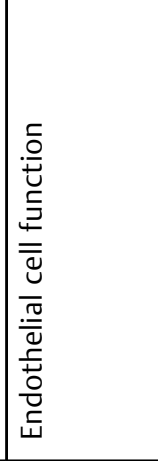 & 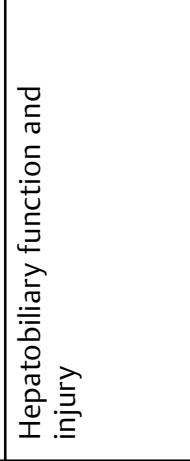 & 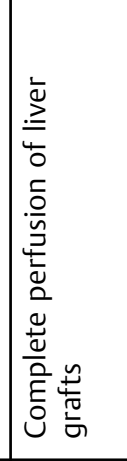 & 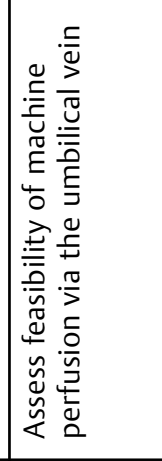 & 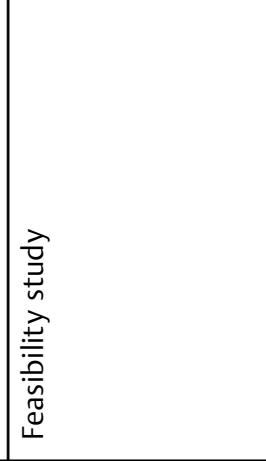 & 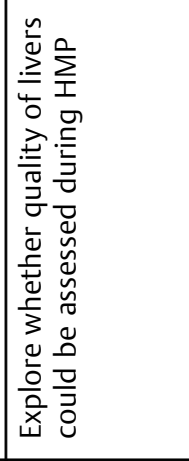 & 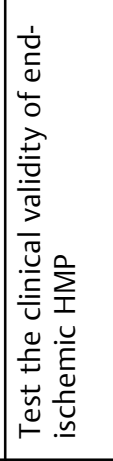 \\
\hline ఏ & & $\nabla$ & $\sim$ & $\sim$ & $\frac{\pi}{C}$ & $\frac{\pi}{C}$ & - & $\stackrel{\Delta}{\sim}$ & $\nabla$ \\
\hline 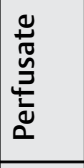 & & $\begin{array}{l}\overline{\tilde{n}} \\
\underline{\underline{\hat{y}}}\end{array}$ & $\sum_{\substack{n \\
\vdots}}^{n}$ & $\sum_{j}^{n}$ & $\begin{array}{l}\overline{\tilde{n}} \\
\underline{\underline{v}}\end{array}$ & $\sum_{\substack{1 \\
\vdots}}^{n}$ & $\begin{array}{l}\overline{\tilde{n}} \\
\underline{\underline{\underline{v}}}\end{array}$ & $\sum_{\substack{1 \\
\vdots}}^{n}$ & $\begin{array}{l}\bar{n} \\
\underline{\hat{n}}\end{array}$ \\
\hline 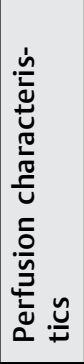 & & 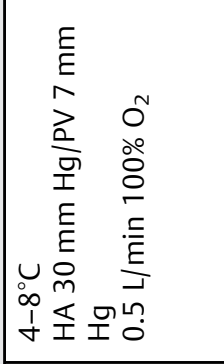 & 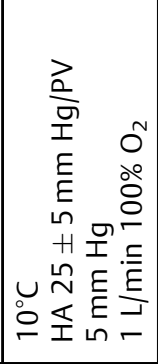 & 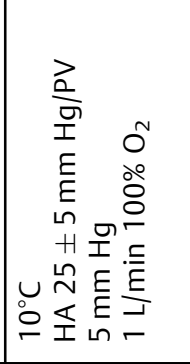 & 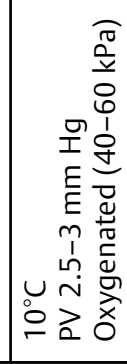 & 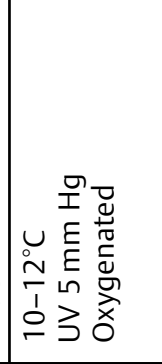 & 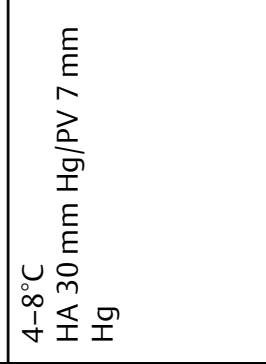 & 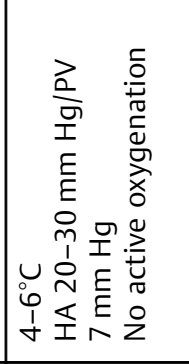 & 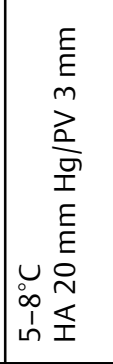 \\
\hline$\hat{\Sigma} \equiv$ & & $\stackrel{\infty}{m}$ & 6 & 0 & $m$ & $m$ & $\simeq$ & $\approx$ & $\stackrel{m}{r}$ \\
\hline 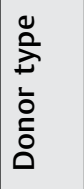 & 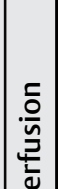 & 商 & 号 & 商 & Ð઼ & ○ે & 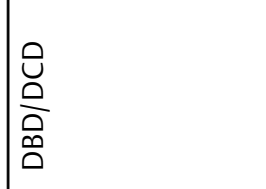 & 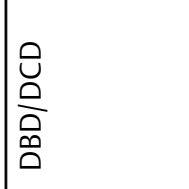 & 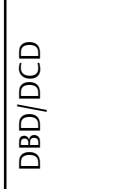 \\
\hline 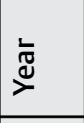 & 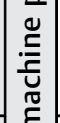 & $\stackrel{\infty}{\grave{\alpha}}$ & 产 & 苘 & 号 & 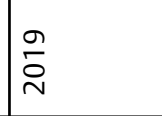 & $\frac{m}{\stackrel{n}{\nu}}$ & $\frac{\sim}{\stackrel{N}{\sim}}$ & $\bar{\Sigma}$ \\
\hline 高 & 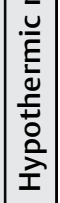 & 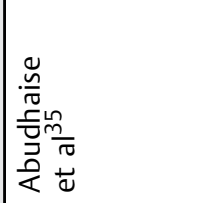 & 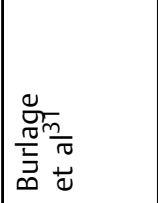 & 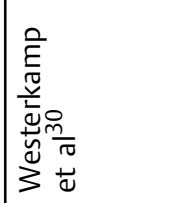 & 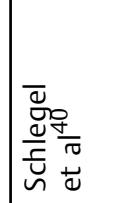 & 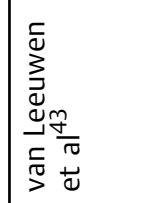 & 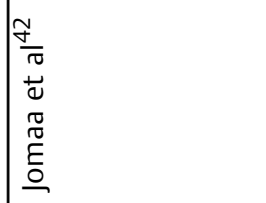 & 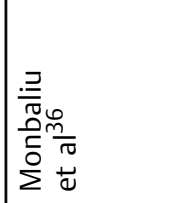 & 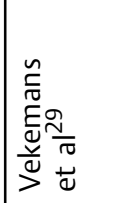 \\
\hline
\end{tabular}




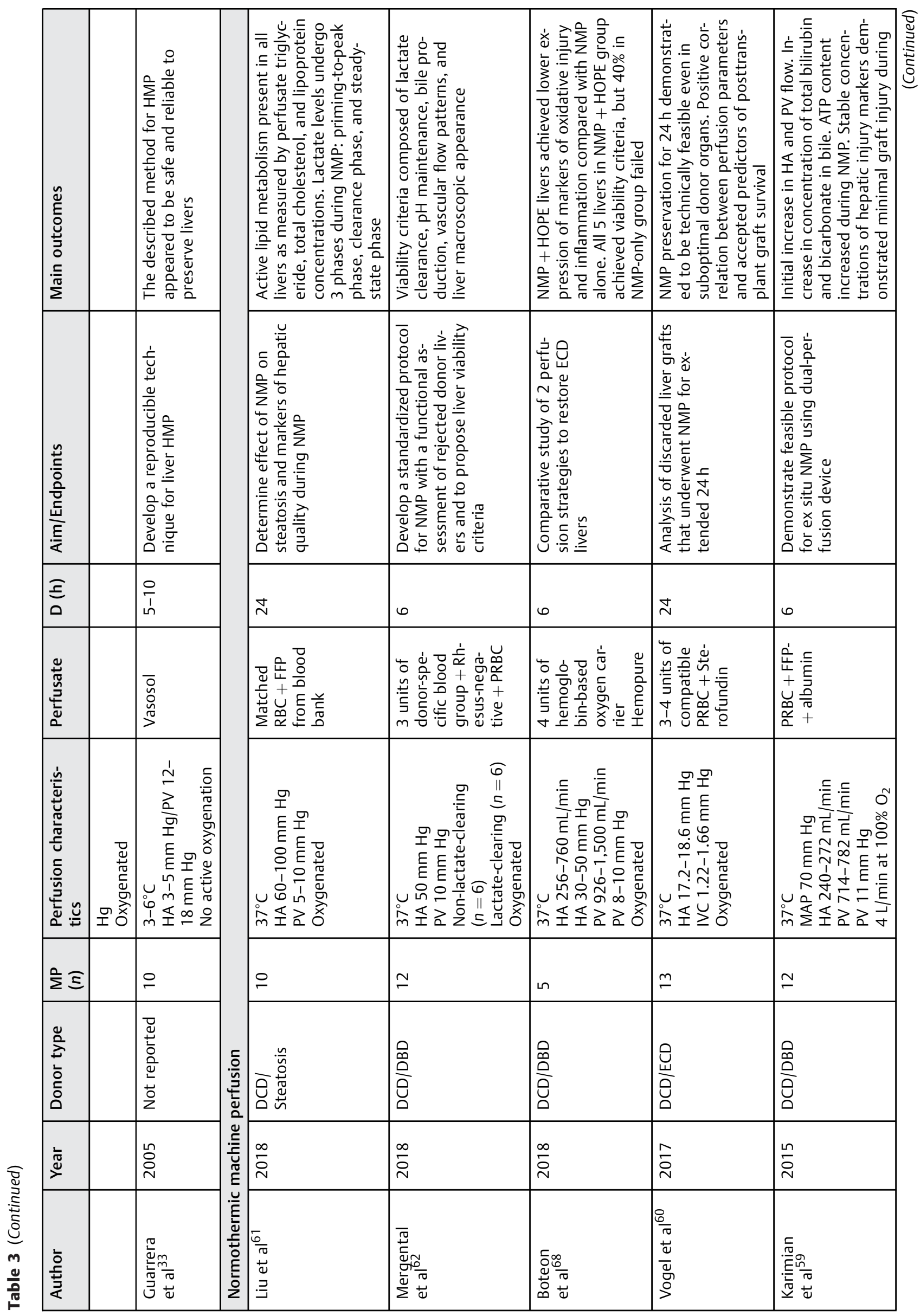




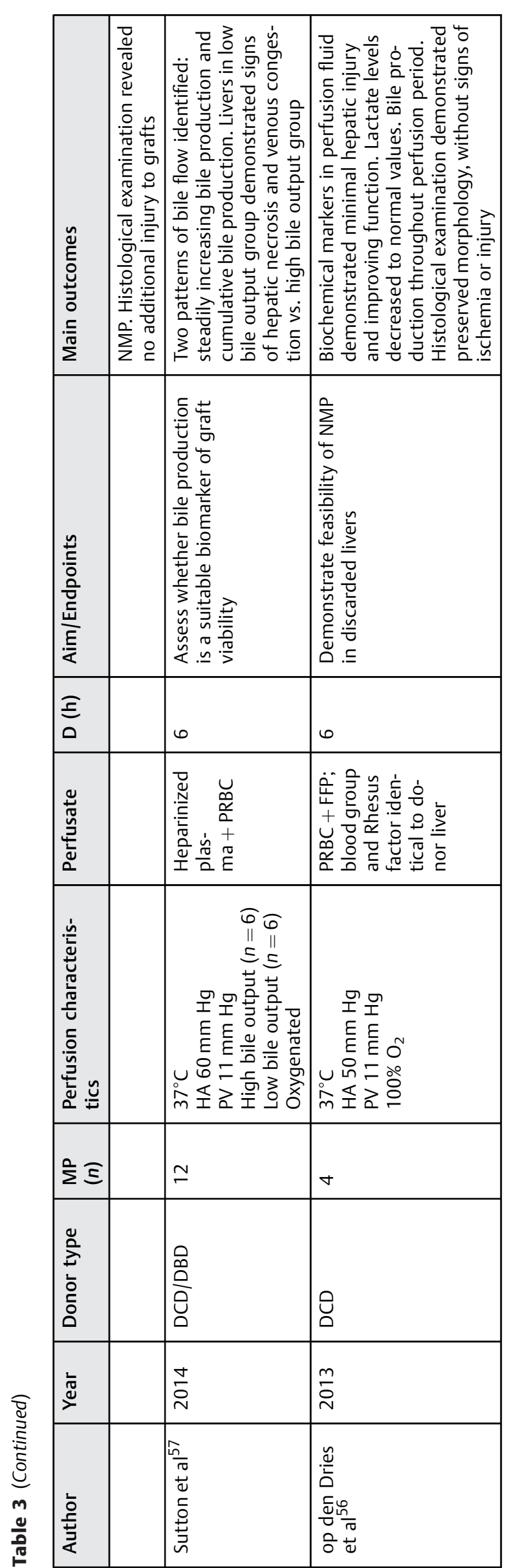

infiltration compared with SCS livers. Together, these results provide mechanistic insights for the understanding of the reduction of IRI provided by perfusion of liver grafts. ${ }^{45,54}$

In all the aforementioned studies, dual perfusion was the perfusion route of choice. Hepatic artery and portal vein flows were around 0.2 to $0.3 \mathrm{~L} / \mathrm{min}$ and 0.8 to $1.25 \mathrm{~L} / \mathrm{min}$, respectively. The duration of NMP varied between the studies ranging from 1.42 to 24 hours with a median time (when described) varying from 8 to 11.5 hours. In two studies, there was a period of initial SCS approximately 4 hours prior to $\mathrm{NMP}^{49,50}$ Red blood cells were the oxygen transporter in all the mentioned studies in association with either Gelofusine or FFP or Steen solution.

Finally, there are eight ongoing clinical trials registered in Clinical Trials.gov that assess the impact of NMP on LT (-Table 2). The Cleveland group is conducting three of them, which use an institutional liver MP device. The number of patients to be recruited varies from 15 to 25 in the studies. In general, the objective is to evaluate the safety and viability of MP to preserve orphaned livers and ECD organs (NCT02515708, NCT04023773, and NCT03456284). Similarly, the Valencia group in Spain is conducting a clinical trial dedicated to evaluate the efficacy of MP of steatotic livers compared with the SCS. The goal is to enroll up to 50 patients, and the final results are expected by 2023 (NCT03930459). Two Canadian groups, both using the OrganOx metra MP device are conducting two other clinical trials. The Alberta group aims to enroll 50 patients and the completion date is estimated in 2021 (NCT03089840). The Toronto group will enroll 40 patients (NCT02478151). Currently, two multicenter clinical trials are being conducted in the United States. Twenty transplant centers participated in one of these studies that uses the TransMedics MP device (TransMedics, Inc.) and initially aimed to enroll 300 participants. Recruitment is already closed and the results are expected by the end of 2020 (NCT02522871). The Duke University group leads the second multicenter trial on a group of 15 transplant centers. This study uses the Metra OrganOx device and aims to enroll 266 participants until December 2020 (NCT02775162). The results of these two large multicenter clinical trials will probably be critical for the approval of NMP devices by the U.S. Food and Drug Administration agency in the United States.

\section{Normothermic Machine Perfusion in Experimental Studies Using Discarded Human Livers}

Similar to what happened in the HMP scenario, experimental animal studies, mainly using porcine models, anticipated studies of NMP with discarded human livers. ${ }^{55}$ Generally, preclinical studies of NMP using discarded livers aimed to verify the feasibility of NMP as a tool to resuscitate graft function, with the objective of identifying biomarkers and/or parameters of organ viability, as well as to investigate the time limit of NMP ( - Table 3). In this way, op den Dries et al demonstrated the viability of NMP in a group of four discarded DCD livers showing a decrease in lactate levels and bile production during the NMP period of 6 hours. ${ }^{56}$ In addition, Sutton et al used a 6-hour NMP protocol after 
6.5 hours of SCS, to show that livers with low bile production have more signs of necrosis and hepatic congestion compared with livers with high bile output. ${ }^{57}$ However, more recent studies underline the importance of bile composition instead of mere bile production. ${ }^{58}$ Karimian et al established a detailed technical protocol for NMP with dual perfusion in 12 ECD livers and Vogel et al showed that within 24 hours of NMP, there is a positive correlation between perfusion parameters and accepted predictors of post-LT graft survival. $^{59,60}$ The Cleveland group evaluated the effect of 24 hours of NMP on lipid metabolism in steatotic livers. They showed active lipid metabolism over time with an increase in triglyceride levels and a decrease in total cholesterol, high-density lipoprotein, and low-density lipoprotein cholesterol, suggesting that MP can be used for lipid modulation. ${ }^{61}$ After a preclinical study that evaluated the viability criteria in 12 ECD livers, Mergental et al reported a series of five transplanted livers with an uneventful postoperative period in all cases. ${ }^{62,63}$ The Cambridge group published about their NMP experiences in two articles where they initially assessed the impact of different $\mathrm{O}_{2}$ tensions in the perfusate during NMP of 12 discarded ECD livers. A high $\mathrm{O}_{2}$ tension was associated with an increased risk of post-reperfusion syndrome and sustained vasoplegia. In total, 47 livers were perfused using NMP, and 20 livers were transplanted from a group of 28 livers accepted for possible transplantation. In addition, 2 livers were transplanted from a group of 19 livers initially declined for transplantation. One recipient died due to PNF and one presented EAD, and three out of four recipients who developed intrahepatic biliary lesions required a retransplantation. ${ }^{64,65}$ Although MP offers the possibility of increasing the group of transplantable livers due to all the advantages mentioned earlier, these results remind us that care must be taken not to push the limits too far when deciding to implant an initially declined liver. Van Leeuwen et al recently proposed a definition of viability criteria based on both hepatocellular (liver parenchyma) and cholangiocyte (bile duct) function (see section "Hypothermic followed by Normothermic Machine Perfusion"). ${ }^{66}$ In addition, we hope that the results of the Viability Testing and Transplantation of marginal Livers (VITTAL study), an ongoing prospective clinical trial, can provide more information on the algorithm to decide which initially discarded liver should be transplanted (NCT02740608; - Table 2). ${ }^{67}$

\section{Hypothermic followed by Normothermic Machine Perfusion}

In a proof-of-concept preclinical work, Boteon et al tested a sequential protocol of 2 hours of HOPE and subsequent 6 hours of NMP, which was compared with NMP alone. The combination of HOPE and NMP leads to a lower expression of oxidative stress markers, with all livers achieving viability criteria against only $40 \%$ of livers in the NMP group. ${ }^{68}$ This strategy, which aims to combine the advantages of HMP to resuscitate the energy levels of the organ and reduce cell injury with the functional evaluation of the organ provided by the NMP, was subsequently explored in the clinical setting by the Groningen group. De Vries et al enrolled 20 discarded livers in a singlearm prospective clinical trial of initial DHOPE followed by an intermediate phase of controlled oxygenated rewarming before the final NMP period (DHOPE-COR-NMP). Seven of the 20 livers were subjected to the DHOPE-COR-NMP protocol, 5 met the LT viability criteria during 2.5 hours of NMP, while 2 livers were declined for transplantation during the NMP evaluation. All transplanted livers reached the primary endpoint of 3-month graft survival. ${ }^{69}$ These results motivated the Groningen group to conduct a national prospective clinical trial where initially declined livers underwent the same DHOPE-COR-NMP protocol using an acellular perfusion solution containing a hemoglobin-based oxygen carrier, HBOC-201 (Hemopure; HbO2 Therapeutics LLC). Sixteen livers were enrolled and $69 \%(11 / 16)$ of them met all the viability criteria, including lactate $<1.7 \mathrm{mmol} / \mathrm{L}$, perfusate $\mathrm{pH} 7.35$ to 7.45 , bile production $>10 \mathrm{~mL}$, and bile $\mathrm{pH}>7.45$ within the first 2.5 hours of NMP. All 11 livers were successfully implanted and the patient and graft survival rates were $100 \%$ at 3 and 6 months. $^{66}$

\section{Logistical Aspects of Machine Perfusion}

Based on the studies reviewed here, we believe that MP is a promising technology for organ rescue and function assessment and improvement. However, its implementation in clinical practice will require caution from the transplant community. Due to logistical and operational reasons, endischemic MP will probably be used in the clinical situation, instead of immediate MP at the donor hospital as happened in some initial clinical trials. Because of its high costs, MP is expected to be reserved for organs at high risk of developing complications of PNF and EAD post-LT, such as ECD organs (e.g., DCD, steatosis, advanced age, expected prolonged CIT, and hemodynamically unstable donors). In addition, MP is time-consuming and requires experienced personnel to set up the machine, place the organ, and monitor the perfusion session. It will probably increase the already overloaded work time of many transplant teams. In general, we believe that the accepting surgical team should be responsible for placement of the organ on the device and its initial monitoring. Special trained organ perfusionists may be responsible for monitoring the organ during MP. Centers might need to establish either a specialized perfusion room or use one of the operating rooms, as cannulation and perfusion of the organ must be performed in a location that meets the current standards for performing sterile procedures. Currently, the cost of MP devices available on the market is around US\$ 100,000 (-Fig. 2). Disposable sets cost from US\$ 5,000 to 30,000 per unit. In general, the additional cost of MP per transplant is estimated between US\$25,000 and 50,000 considering the device, disposable, personnel, blood products, increased donor operation room time, etc. It raises the question of who will be responsible for these extra costs: the transplant center that pumps the organ, or in case that the organ is perfused by the allocation agency (e.g., OPO in the United States and Eurotransplant in Europe) should the extra costs be shared by all centers under its jurisdiction? Would 


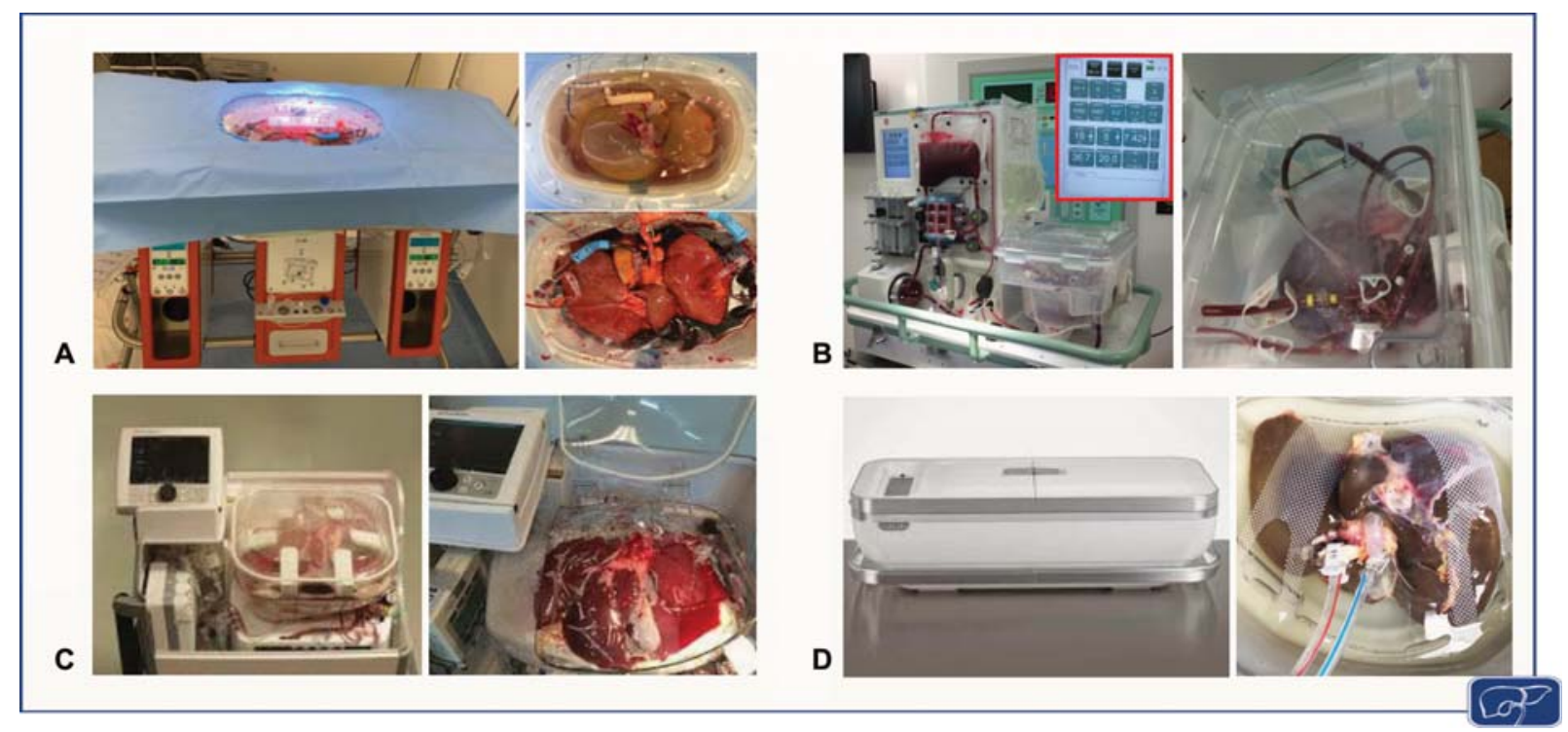

Fig. 2 Panel showing four of the currently available devices available in the market for ex situ liver perfusion. (A) Left: an overview of the Liver Assist machine perfusion device (Organ Assist Products, B.V., Groningen, NL); top right: single hypothermic machine perfusion with a commercial perfusion solution; bottom right: dual normothermic machine perfusion using a perfusion solution based on a hemoglobin-based oxygen carrier. (B) Left: an overview of the OrganOx metra machine perfusion device (OrganOx Limited, Oxford, UK), with a close-up on the perfusion parameters panel; right: a closed container with a liver being perfused using red blood cells as perfusate. (C) Left: an overview on the TransMedics Organ Care System (OCS) Liver (Transmedics Inc, Andover, MA) machine perfusion device; right: close-up of a liver being perfused with red blood cells as a perfusate. (D) Left: an overview of the LiverPort machine perfusion device (Organ Recovery Systems, Itasca, IL), right: dual hypothermic machine perfusion with a commercial perfusion solution.

this additional cost be covered by all medical insurances? These questions and many others raised by the future implementation of MP in clinical practice were highlighted by a committee of experts on organ perfusion and published by Quintini et al in 2018 on behalf of the American Society of Transplant Surgeons. As indicated, the answers to all questions will require the participation of the entire liver transplant community, including transplant centers, allocation agencies, medical insurance organizations, and scientific associations. ${ }^{70}$

\section{Pitfalls in Machine Preservation Studies}

As with any clinical trial, it is extremely important to avoid selection biases. This is particularly important for organ transplantation, as both characteristics of recipient and the graft can have a tremendous impact on outcomes. It is very important to randomize the groups and the randomization process needs to be done after the organ has been accepted and not at the time of the donor offer. Although attempts are made, there are currently no widely accepted criteria for transplantability of a liver graft. Unconscious biases may influence the decision to accept or decline an organ at the time of the procurement. To transplant grafts that were initially declined by all centers in the region but later successfully transplanted after MP does not necessarily mean that those organs were nonviable prior to MP-pointing to several studies showing good outcomes for "liversthat-nobody-wants." ${ }^{25,71-75}$ Another limitation for clinical trials of MP is that it requires large sample sizes (and increased costs) for variables that are infrequent like PNF and ischemic cholangiopathy. Therefore, it seems more rea- sonable to perform clinical trials using ECD organs, like DCD and severely steatotic organs, until more data are available.

It is also very important to choose the right endpoints when designing clinical trials. ${ }^{76-78}$ The primary endpoint needs to be of significant clinical relevance. A surrogate endpoint has been defined as "a biomarker that is intended to substitute for a clinical endpoint" and generally is considered valid given a more rapid and frequent incidence and strong association with traditional endpoints. ${ }^{77}$ Obtaining statistical significance for irrelevant endpoints does not mean superiority for MP. For example, peak transaminase posttransplant has been used in several studies and even as the primary endpoint in a RCT. ${ }^{48}$ We believe peak transaminases/EAD should not be used as primary endpoint in machine preservation trials for two reasons..$^{20}$ First, transaminases have not been shown to have an important clinical significance in both the liver donor and post-LT settings, unless extremely high. ${ }^{79-83}$ Second, since the grafts in the MP trials are flushed with several extra liters of preservation solution, part of the transaminases accumulated in the liver before transplantation are eliminated, and they have a long half-life $(17 \pm 5$ hours for AST, $47 \pm 10$ hours for ALT). ${ }^{84}$ Therefore, if transaminases are going to be used as secondary endpoints, ideally the grafts from the control arm (standard cold preservation) should be flushed with the same amount of fluid used in the perfusion circuit just before implantation to minimize the differences related to the wash-out phenomenon.

\section{Conclusion}

Over the last decade, MP of donor livers has gained considerable interest as an approach to resuscitate and assess ECD 
livers prior to transplantation. After extensive preclinical work, prospective clinical trials increased the evidence of the beneficial effects of MP over SCS preservation. It is still early to definitively confirm that MP is superior to SCS preservation in regard to graft and patient survival. We need to wait for more RCTs comparing MP with SCS preservation, as well as comparing different modalities of machine preservation. Likely, all modalities of MP could have a role in the clinical setting with each having pros and cons in different situations. There are grounds for optimism that MP leads to better preservation of the biliary tree with less expected ischemic cholangiopathy. Besides, there has been a lot of progress toward optimizing MP in terms of duration, perfusion solution, and temperatures used. Within the area of liver MP, we are now progressing toward a more individualized approach based on donor organ characteristics. In the coming years, it should become clear which organ would benefit most from MP and under which preservation conditions (e.g., hypothermic, normothermic, or both). The sequential strategy of HMP followed by NMP seems to combine the advantages of both protocols, the restoration of cellular energy with the possibility to evaluate organ function, and providing conditions for organ modulation and recondition. As such, genetic modulation therapies can be administered during MP to improve postLT outcome. ${ }^{85-88}$ In the future, the development of nanoparticles may be translated into clinical use with the potential to mitigate organ damage through a variety of mechanisms. ${ }^{89}$ More studies are needed to address these strategies. On the other hand, important logistical issues related to costs, MP reimbursement, personnel in charge, time, and place of the MP session must be addressed to move MP from the research environment to a reality in the clinical practice of LT. Altogether, we can expect that, in the coming years, the field of liver preservation will become even more interesting with new technologies and more evidence through clinical trials.

\section{Main Concepts and Learning Points}

- Machine perfusion is a feasible and safe procedure for organ preservation.

- Hypothermic oxygenated machine perfusion effectively restores cellular energetic capital within few hours of perfusion.

- Normothermic machine perfusion may be more adequate to assess organ viability during the perfusion session.

- Further clinical trials are required to define in which clinical scenario MP will be superior to SCS and costeffective.

\section{Authors' Contributions}

E.B-R., I.M.A.B., J.B., S.I., and P.N.M. contributed to conception and design, acquisition of data, and analysis and interpretation of data. E.B.-R., I.M.A.B., and P.N.M. drafted the article and revised it critically for important intellectual content. P.N.M. approved the final version to be published.

\section{Conflicts of Interest}

None.

\section{References}

1 Mazzaferro V, Battiston C, Sposito C. Pro (with caution): extended oncologic indications in liver transplantation. Liver Transpl 2018; 24(01):98-103

2 Schaefer B, Zoller H, Schneeberger S. Con: liver transplantation for expanded criteria malignant diseases. Liver Transpl 2018;24(01): 104-111

3 Kim WR, Lake JR, Smith JM, et al. OPTN/SRTR 2017 annual data report: liver. Am J Transplant 2019;19(Suppl 2):184-283

4 Neuberger J. Liver transplantation in the United Kingdom. Liver Transpl 2016;22(08):1129-1135

5 Saidi RF. Utilization of expanded criteria donors in liver transplantation. Int J Organ Transplant Med 2013;4(02):46-59

6 Lindbergh CA. An apparatus for the culture of whole organs. J Exp Med 1935;62(03):409-431

7 Dutkowski P, de Rougemont O, Clavien PA. Alexis Carrel: genius, innovator and ideologist. Am J Transplant 2008;8(10):19982003

8 Brettschneider L, Daloze PM, Huguet C, et al. Successful orthotopic transplantation of liver homografts after eight to twentyfive hours preservation. Surg Forum 1967;18:376-378

9 Kestens PJ, Otte JB, Alexandre GP, et al. [Human orthotopic liver graft]. Acta Chir Belg 1970;69(05):285-303

10 Collins GM, Bravo-Shugarman M, Terasaki PI. Kidney preservation for transportation. Initial perfusion and 30 hours' ice storage. Lancet 1969;2(7632):1219-1222

11 Belzer FO, D'Alessandro AM, Hoffmann RM, et al. The use of UW solution in clinical transplantation. A 4-year experience. Ann Surg 1992;215(06):579-583, discussion 584-585

12 Laing RW, Mergental H, Mirza DF. Normothermic ex-situ liver preservation: the new gold standard. Curr Opin Organ Transplant 2017;22(03):274-280

13 Martins PN, Selzner M, Dayangac M, et al. What is hot and new in basic science in liver transplantation in 2018? report of the Basic Science Committee of the International Liver Transplantation Society. Transplantation 2019;103(04):654-659

14 Taner T, Martins PN, Ling Q et al. What is hot and new in basic and translational science in liver transplantation in 2019? Report of the Basic and Translational Research Committee of the International Liver Transplantation Society. Transplantation 2019;103 (04):654-659

15 Karangwa SA, Dutkowski P, Fontes P, et al. Machine perfusion of donor livers for transplantation: a proposal for standardized nomenclature and reporting guidelines. Am J Transplant 2016; 16(10):2932-2942

16 Bruinsma BG, Berendsen TA, Izamis ML, Yeh H, Yarmush ML, Uygun K. Supercooling preservation and transplantation of the rat liver. Nat Protoc 2015;10(03):484-494

17 de Vries RJ, Tessier SN, Banik PD, et al. Supercooling extends preservation time of human livers. Nat Biotechnol 2019;37(10): 1131-1136

18 von Horn C, Minor T. Transient hyperthermia during oxygenated rewarming of isolated rat livers. Transpl Int 2020;33(03): 272-278

19 Schlegel A, de Rougemont O, Graf R, Clavien PA, Dutkowski P. Protective mechanisms of end-ischemic cold machine perfusion in DCD liver grafts. J Hepatol 2013;58(02):278-286

20 Dutkowski P, Guarrera JV, de Jonge J, Martins PN, Porte RJ, Clavien PA. Evolving trends in machine perfusion for liver transplantation. Gastroenterology 2019;156(06):1542-1547

21 De Carlis R, Lauterio A, Ferla F, Di Sandro S, Sguinzi R, De Carlis L. Hypothermic machine perfusion of liver grafts can safely extend cold Ischemia for Up to 20 hours in cases of necessity. Transplantation 2017;101(07):e223-e224 
22 Muller X, Schlegel A, Kron P, et al. Novel real-time prediction of liver graft function during hypothermic oxygenated machine perfusion before liver transplantation. Ann Surg 2019;270(05): 783-790

23 Guarrera JV, Henry SD, Samstein B, et al. Hypothermic machine preservation in human liver transplantation: the first clinical series. Am J Transplant 2010;10(02):372-381

24 De Deken J, Kocabayoglu P, Moers C. Hypothermic machine perfusion in kidney transplantation. Curr Opin Organ Transplant 2016;21(03):294-300

25 Guarrera JV, Henry SD, Samstein B, et al. Hypothermic machine preservation facilitates successful transplantation of "orphan" extended criteria donor livers. Am J Transplant 2015;15(01): 161-169

26 Op den Dries S, Sutton ME, Karimian N, et al. Hypothermic oxygenated machine perfusion prevents arteriolonecrosis of the peribiliary plexus in pig livers donated after circulatory death. PLoS One 2014;9(02):e88521

27 Dutkowski P, Polak WG, Muiesan P, et al. First comparison of hypothermic oxygenated perfusion versus static cold storage of human donation after cardiac death liver transplants: an international-matched case analysis. Ann Surg 2015;262(05):764-770, discussion 770-771

28 van Rijn R, Karimian N, Matton APM, et al. Dual hypothermic oxygenated machine perfusion in liver transplants donated after circulatory death. Br J Surg 2017;104(07):907-917

29 Vekemans K, van Pelt J, Komuta M, et al. Attempt to rescue discarded human liver grafts by end ischemic hypothermic oxygenated machine perfusion. Transplant Proc 2011;43(09):3455-3459

30 Westerkamp AC, Karimian N, Matton AP, et al. Oxygenated hypothermic machine perfusion after static cold storage improves hepatobiliary function of extended criteria donor livers. Transplantation 2016;100(04):825-835

31 Burlage LC, Karimian N, Westerkamp AC, et al. Oxygenated hypothermic machine perfusion after static cold storage improves endothelial function of extended criteria donor livers. HPB (Oxford) 2017;19(06):538-546

32 Brüggenwirth IMA, van Leeuwen $\mathrm{OB}$, de Vries Y, et al. Extended hypothermic oxygenated machine perfusion enables ex situ preservation of porcine livers for up to 24 hours. JHEP Rep 2020;2(02): 100092

33 Guarrera JV, Estevez J, Boykin J, et al. Hypothermic machine perfusion of liver grafts for transplantation: technical development in human discard and miniature swine models. Transplant Proc 2005;37(01):323-325

34 Schlegel A, Kron P, Dutkowski P. Hypothermic oxygenated liver perfusion: basic mechanisms and clinical application. Curr Transplant Rep 2015;2(01):52-62

35 Abudhaise H, Davidson BR, DeMuylder P, Luong TV, Fuller B. Evolution of dynamic, biochemical, and morphological parameters in hypothermic machine perfusion of human livers: a proofof-concept study. PLoS One 2018;13(09):e0203803

36 Monbaliu D, Liu Q, Libbrecht L, et al. Preserving the morphology and evaluating the quality of liver grafts by hypothermic machine perfusion: a proof-of-concept study using discarded human livers. Liver Transpl 2012;18(12):1495-1507

37 Lüer B, Koetting M, Efferz P, Minor T. Role of oxygen during hypothermic machine perfusion preservation of the liver. Transpl Int 2010;23(09):944-950

38 Schlegel A, Dutkowski P. Role of hypothermic machine perfusion in liver transplantation. Transpl Int 2015;28(06):677-689

39 Debbaut C, Monbaliu D, Casteleyn C, et al. From vascular corrosion cast to electrical analog model for the study of human liver hemodynamics and perfusion. IEEE Trans Biomed Eng 2011;58 (01):25-35

40 Schlegel A, Kron P, De Oliveira ML, Clavien PA, Dutkowski P. Is single portal vein approach sufficient for hypothermic machine perfusion of DCD liver grafts? J Hepatol 2016;64(01):239-241
41 Brüggenwirth IMA, Burlage LC, Porte RJ, Martins PN. Is single portal vein perfusion the best approach for machine preservation of liver grafts? J Hepatol 2016;64(05):1194-1195

42 Jomaa A, Gurusamy K, Siriwardana PN, et al. Does hypothermic machine perfusion of human donor livers affect risks of sinusoidal endothelial injury and microbial infection? A feasibility study assessing flow parameters, sterility, and sinusoidal endothelial ultrastructure. Transplant Proc 2013;45(05):1677-1683

43 van Leeuwen OB, Fujiyoshi M, Ubbink R, et al. Ex situ machine perfusion of human donor livers via the surgically reopened umbilical vein: a proof of concept. Transplantation 2019;103 (10):2130-2135

44 Jain S, Xu H, Duncan H, et al. Ex-vivo study of flow dynamics and endothelial cell structure during extended hypothermic machine perfusion preservation of livers. Cryobiology 2004;48(03):322-332

45 Ravikumar R, Jassem W, Mergental H, et al. Liver transplantation after ex vivo normothermic machine preservation: a Phase 1 (first-in-man) clinical trial. Am J Transplant 2016;16(06): 1779-1787

46 Selzner M, Goldaracena N, Echeverri J, et al. Normothermic ex vivo liver perfusion using Steen solution as perfusate for human liver transplantation: first North American results. Liver Transpl 2016; 22(11):1501-1508

47 Bral M, Gala-Lopez B, Bigam D, et al. Preliminary single-center Canadian experience of human normothermic ex vivo liver perfusion: results of a clinical trial. Am J Transplant 2017;17(04): 1071-1080

48 Nasralla D, Coussios CC, Mergental H, et al; Consortium for Organ Preservation in Europe. A randomized trial of normothermic preservation in liver transplantation. Nature 2018;557 (7703):50-56

49 Ghinolfi D, Rreka E, De Tata V, et al. Pilot, open, randomized, prospective trial for normothermic machine perfusion evaluation in liver transplantation from older donors. Liver Transpl 2019;25 (03):436-449

50 Liu Q Hassan A, Pezzati D, et al. Ex-situ liver machine perfusion: the impact of fresh frozen plasma. Liver Transpl 2020;26(02): 215-226

51 van Leeuwen $\mathrm{OB}$, de Meijer VE, Porte RJ. Viability criteria for functional assessment of donor livers during normothermic machine perfusion. Liver Transpl 2018;24(10):1333-1335

52 Perera T, Mergental H, Stephenson B, et al. First human liver transplantation using a marginal allograft resuscitated by normothermic machine perfusion. Liver Transpl 2016;22(01): 120-124

53 Watson CJ, Kosmoliaptsis V, Randle LV, et al. Preimplant normothermic liver perfusion of a suboptimal liver donated after circulatory death. Am J Transplant 2016;16(01):353-357

54 Jassem W, Xystrakis E, Ghnewa YG, et al. Normothermic machine perfusion (NMP) inhibits proinflammatory responses in the liver and promotes regeneration. Hepatology 2019;70(02):682-695

55 Marecki H, Bozorgzadeh A, Porte RJ, Leuvenink HG, Uygun K, Martins PN. Liver ex situ machine perfusion preservation: a review of the methodology and results of large animal studies and clinical trials. Liver Transpl 2017;23(05):679-695

56 op den Dries S, Karimian N, Sutton ME, et al. Ex vivo normothermic machine perfusion and viability testing of discarded human donor livers. Am J Transplant 2013;13(05):1327-1335

57 Sutton ME, op den Dries S, Karimian N, et al. Criteria for viability assessment of discarded human donor livers during ex vivo normothermic machine perfusion. PLoS One 2014;9(11):e110642

58 Matton APM, de Vries Y, Burlage LC, et al. Biliary Bicarbonate, $\mathrm{pH}$, and glucose are suitable biomarkers of biliary viability during ex situ normothermic machine perfusion of human donor livers. Transplantation 2019;103(07):1405-1413

59 Karimian N, Matton AP, Westerkamp AC, et al. Ex situ normothermic machine perfusion of donor livers. J Vis Exp 2015;(99): e52688 
60 Vogel T, Brockmann JG, Quaglia A, et al. The 24-hour normothermic machine perfusion of discarded human liver grafts. Liver Transpl 2017;23(02):207-220

61 Liu Q, Nassar A, Buccini L, et al. Lipid metabolism and functional assessment of discarded human livers with steatosis undergoing 24 hours of normothermic machine perfusion. Liver Transpl 2018;24(02):233-245

62 Mergental H, Stephenson BTF, Laing RW, et al. Development of clinical criteria for functional assessment to predict primary nonfunction of high-risk livers using normothermic machine perfusion. Liver Transpl 2018;24(10):1453-1469

63 Mergental H, Perera MT, Laing RW, et al. Transplantation of declined liver allografts following normothermic ex-situ evaluation. Am J Transplant 2016;16(11):3235-3245

64 Watson CJE, Kosmoliaptsis V, Randle LV, et al. Normothermic perfusion in the assessment and preservation of declined livers before transplantation: hyperoxia and vasoplegia-important lessons from the first 12 cases. Transplantation 2017;101(05): 1084-1098

65 Watson CJE, Kosmoliaptsis V, Pley C, et al. Observations on the ex situ perfusion of livers for transplantation. Am J Transplant 2018; 18(08):2005-2020

66 van Leeuwen OB, de Vries Y, Fujiyoshi M, et al. Transplantation of high-risk donor livers after ex situ resuscitation and assessment using combined hypo- and normothermic machine perfusion: a prospective clinical trial. Ann Surg 2019;270(05):906-914

67 Laing RW, Mergental H, Yap C, et al. Viability testing and transplantation of marginal livers (VITTAL) using normothermic machine perfusion: study protocol for an open-label, nonrandomised, prospective, single-arm trial. BMJ Open 2017;7 (11):e017733

68 Boteon YL, Laing RW, Schlegel A, et al. Combined hypothermic and normothermic machine perfusion improves functional recovery of extended criteria donor livers. Liver Transpl 2018;24(12): 1699-1715

69 de Vries Y, Matton APM, Nijsten MWN, et al. Pretransplant sequential hypo- and normothermic machine perfusion of suboptimal livers donated after circulatory death using a hemoglobin-based oxygen carrier perfusion solution. Am J Transplant 2019;19(04):1202-1211

70 Quintini C, Martins PN, Shah S, et al; American Society of Transplant Surgeons Standards Committee. Implementing an innovated preservation technology: the American Society of Transplant Surgeons' (ASTS) Standards Committee White Paper on Ex Situ Liver Machine Perfusion. Am J Transplant 2018;18(08):1865-1874

71 Carpenter DJ, Chiles MC, Verna EC, et al. Deceased brain dead donor liver transplantation and utilization in the United States: nighttime and weekend effects. Transplantation 2019;103(07): 1392-1404

72 Sotiropoulos GC, Lang H, Saner FH, et al. Long-term results after liver transplantation with "livers that nobody wants" within Eurotransplant: a center's experience. Transplant Proc 2008;40 (09):3196-3197

73 McCormack L, Quiñonez E, Ríos MM, et al. Rescue policy for discarded liver grafts: a single-centre experience of trans- planting livers 'that nobody wants'. HPB (Oxford) 2010;12(08): 523-530

74 Tector AJ, Mangus RS, Chestovich P, et al. Use of extended criteria livers decreases wait time for liver transplantation without adversely impacting posttransplant survival. Ann Surg 2006;244 (03):439-450

75 Halazun KJ, Quillin RC, Rosenblatt R, et al. Expanding the margins: high volume utilization of marginal liver grafts among $>2000$ liver transplants at a single institution. Ann Surg 2017;266(03): 441-449

76 Muller X, Marcon F, Sapisochin G, et al. Defining benchmarks in liver transplantation: a multicenter outcome analysis determining best achievable results. Ann Surg 2018;267(03):419-425

77 Stevens LA, Greene T, Levey AS. Surrogate end points for clinical trials of kidney disease progression. Clin J Am Soc Nephrol 2006;1 (04):874-884

78 Agopian VG, Harlander-Locke MP, Markovic D, et al. Evaluation of early allograft function using the liver graft assessment following transplantation risk score model. JAMA Surg 2018;153(05): 436-444

79 Rosen HR, Martin P, Goss J, et al. Significance of early aminotransferase elevation after liver transplantation. Transplantation 1998; 65(01):68-72

80 Feng S, Goodrich NP, Bragg-Gresham JL, et al. Characteristics associated with liver graft failure: the concept of a donor risk index. Am J Transplant 2006;6(04):783-790

81 Radunz S, Paul A, Nowak K, Treckmann JW, Saner FH, Mathé Z. Liver transplantation using donor organs with markedly elevated liver enzymes: how far can we go? Liver Int 2011;31(07): 1021-1027

82 Gadsden MM, Goldberg DS. Hepatic dysfunction in deceased donors in the age of the opioid epidemic. Transplantation 2018; 102(10):e403

83 Martins PN, Rawson A, Movahedi B, et al. Single-center experience with liver transplant using donors with very high transaminase levels. Exp Clin Transplant 2019;17(04):498-506

84 Dufour DR, Lott JA, Nolte FS, Gretch DR, Koff RS, Seeff LB. Diagnosis and monitoring of hepatic injury. II. Recommendations for use of laboratory tests in screening, diagnosis, and monitoring. Clin Chem 2000;46(12):2050-2068

85 Thijssen MF, Brüggenwirth IMA, Gillooly A, Khvorova A, Kowalik TF, Martins PN. Gene Silencing With siRNA (RNA Interference): a new therapeutic option during ex vivo machine liver perfusion preservation. Liver Transpl 2019;25(01):140-151

86 Gillooly AR, Perry J, Martins PN. First Report of siRNA Uptake (for RNA Interference) during ex vivo hypothermic and normothermic liver machine perfusion. Transplantation 2019;103(03):e56-e57

87 Bruggenwirth IMA, Martins PN. RNA interference therapeutics in organ transplantation: The dawn of a new era. Am J Transplant 2020;20(04):931-941

88 Buchwald JE, Xu J, Bozorgzadeh A, Martins PN. Therapeutics administered during ex vivo liver machine perfusion: An overview. World J Transplant 2020;10(01):1-14

89 Yao CG, Martins PN. Nanotechnology Applications in Transplantation Medicine. Transplantation 2020;104(04):682-693 